\title{
Kallistatin correlates with inflammation in abdominal aortic aneurysm and suppresses its formation in mice
}

\author{
Yuchen $\mathrm{He}^{1}$, Yanshuo $\mathrm{Han}^{2}$, Jia Xing ${ }^{3}$, Xiaoyue Zhai ${ }^{3}$, Shiyue Wang ${ }^{1}$, Shijie Xin ${ }^{1}$, Jian Zhang ${ }^{1}$ \\ ${ }^{1}$ Department of Vascular Surgery, The First Hospital of China Medical University, Shenyang 110001, China; ${ }^{2}$ Department of General Surgery, \\ Shengjing Hospital of China Medical University, Shenyang 110004, China; ${ }^{3}$ Department of Histology and Embryology, China Medical University, \\ Shenyang 110122, China \\ Contributions: (I) Conception and design: Y He, J Xing, Y Han; (II) Administrative support: J Zhang, S Xin, X Zhai; (III) Provision of study \\ materials or patients: Y He, S Wang; (IV) Collection and assembly of data: Y He, S Wang; (V) Data analysis and interpretation: Y He, S Wang; (VI) \\ Manuscript writing: All authors; (VII) Final approval of manuscript: All authors. \\ Correspondence to: Jian Zhang, MD, PhD. Department of Vascular Surgery, The First Hospital of China Medical University, 155\# Nanjing Street, \\ Shenyang 110001, China. Email: jianzhang@cmu.edu.cn.
}

Background: Kallistatin (KS), encoded by SERPINA4, was suggested to play a protective role in many cardiovascular diseases. However, its role in the pathogenesis of abdominal aortic aneurysm (AAA) remains unclear. The aim of this study was to examine the potential association of KS with AAA pathogenesis.

Methods: We examined KS (SERPINA4) expression in human AAA by PCR, immunohistochemistry, western blotting, and enzyme-linked immunosorbent assay (ELISA) and analyzed correlations between kallistain and clinical data. We then analyzed the effect of recombinant KS on AAA formation and the Wingless (Wnt) signaling pathway in a mouse AAA model developed by angiotensin II (AngII) infusion to apolipoprotein E-deficient $\left(\mathrm{ApoE}^{-/-}\right)$mice.

Results: In AAA tissue samples, KS was significantly increased compared with samples from the control group ( $\mathrm{P}<0.001, \mathrm{P}<0.001$, respectively). Clinically, decreased SERPINA4 expression in AAA tissue samples represented an increased rate of iliac artery aneurysm [odds ratio (OR): 0.017; P=0.040]. And decreased plasma KS level represented a high risk for rupture (OR: 0.837; $\mathrm{P}=0.034)$. KS inhibited AAA formation and blocked the Wnt signaling pathway in AngII-infused $\mathrm{ApoE}^{-/-}$mice.

Conclusions: The present study demonstrates that aberrant changes in KS expression occur in AAA. KS plays an important anti-inflammatory role and showed important clinical correlations in AAA. Decreased KS (SERPINA4) level is a risk factor of AAA rupture. Our pre-clinical animal experiments indicate that treatment with recombination KS suppresses AngII-induced aortic aneurysm formation and might be a new target for the drug therapy of AAA.

Keywords: Abdominal aortic aneurysm (AAA); serine proteinase inhibitors (SERPINs); inflammation

Submitted Sep 14, 2019. Accepted for publication Nov 27, 2019.

doi: $10.21037 / \mathrm{cdt} .2019 .12 .08$

View this article at: http://dx.doi.org/10.21037/cdt.2019.12.08

\section{Introduction}

Abdominal aortic aneurysms (AAAs) are defined as the permanent dilation of the abdominal aortic wall beyond a maximum diameter of $>3 \mathrm{~cm}$, or exceeding the standard vessel diameter by $50 \%$ (1). In western countries, $1.3 \%$ mortality over the age of 65 was shown to be caused by
AAA. AAA is affected by a variety of risk factors, including age, gender, lipoprotein levels, smoking, hypertension, and family history, and has a complex pathogenesis $(2,3)$. Inflammation is a critical initial factor in the progression of AAA. Many studies have focused on the impact of inflammatory responses and the immune system on AAA progression (2). In clinical practice, imaging 
techniques and blood parameters were used to evaluate inflammatory changes in AAA. Previous studies reported that inflammatory changes in AAA were associated with its growth and rupture (4).

Zhou and colleagues (5) reported that kallistatin (KS) was significantly decreased in postsurgical sera compared with pre-surgical sera from AAA patients according to their serum proteomics result. KS, encoded by SERPINA4, is a member of the serine proteinase inhibitor (SERPIN) family. KS regulates several signaling pathways and biological functions via two crucial structural elements, an active site, and heparin-binding domain, and has direct vascular effects (6-11). Additionally, Li and colleagues (6) proposed a hypothesis that KS has an important role in AAAs. However, the potential role of KS in AAA has not been proved.

The Wingless (Wnt) signaling pathway is involved in AAA pathogenesis by mediating a variety of cellular activities including proliferation, apoptosis, migration, inflammatory responses, and differentiation $(12,13)$. Many factors associated with inflammation and angiogenesis, including vascular endothelial growth factor (VEGF), intercellular adhesion molecule (ICAM)-1, and tumor necrosis factor (TNF)- $\alpha$, are regulated through the Wnt signaling pathway (14). Moreover, evidence suggests that KS competes with Wnt via its heparin-binding domain for binding to the cell surface co-receptor, lowdensity lipoprotein receptor-related protein 6 (LRP6) to block Wnt canonical signaling, thereby antagonizing the biological effects (15). This hypothesizes that KS might play a protective role in AAA by inhibiting activation of the Wnt signal pathway. Nevertheless, there is no evidence to support this hypothesis.

In this study, we focus on the potential anti-inflammatory role of KS in AAA. We analyzed the expression of KS in plasma, peripheral blood mononuclear cells (PBMCs), and aortic walls of AAA patients and controls, and its association with clinical data, for example, rupture. We examined KS expression in individual cell types within AAA, and found that KS expression correlated with inflammatory genes and Wnt signaling in AAA. We also established angiotensin II (AngII)-infused apolipoprotein E-deficient $\left(\mathrm{ApoE}^{--}\right)$mice as a model of AAA, and detected the protective role of KS in this AAA mouse model. Our results provide compelling evidence for a role of KS in AAA and its potential as a novel therapeutic strategy for the management and treatment of AAA.

\section{Methods}

\section{Participants and sample collection}

Participants gave written informed consent before the study, and the study protocol was approved by the Ethics Committee of the First Hospital of China Medical University, in accordance with the Declaration of Helsinki.

From January 2011 to December 2018, our team collected AAA tissue samples and matched peripheral blood samples, and the corresponding clinical data of 36 consecutive patients from the Department of Vascular Surgery of the First Hospital of China Medical University. Computed tomography angiography (CTA) was also performed for the diagnosis of AAA. All 36 AAA patients undergoing the elective open surgery were eligible for inclusion in the current study.

Inclusion criteria for the AAA group included sufficient quality of AAA tissue samples and blood samples and the availability of corresponding patient history and clinical data, including demographic and clinical characteristics, symptoms, and blood parameters. Exclusion criteria for the AAA group included Ehlers-Danlos syndrome, Marfan syndrome, other known vascular or connective tissue disorders, cancer, infection, and any other immune-related disease.

AAA tissue samples were collected using standard procedures from the anterior sac of the infrarenal abdominal aorta and divided into two parts: one was stored in liquid nitrogen, and the other was fixed in $4 \%$ paraformaldehyde for paraffin sections.

Twelve healthy aortic tissue samples obtained from organ donors in Department of Transplant Surgery and matched with 36 AAA tissue samples were used as controls. Exclusion criteria for the control group included cancer, infection, or any other immune-related disease that may influence the study.

Venipuncture was performed with written informed consent of the participants, who were aware of the intended use of the samples, and the Ethics Committee of the First Hospital of China Medical University approved the venous blood collection protocol in accordance with the declaration of Helsinki. Venipuncture was performed after a 12-h fast and specimens were collected in ethylenediaminetetraacetic (EDTA) plastic tubes (BD Vacutainer ${ }^{\circledR}$ lavender, $5.0 \mathrm{~mL}$ ).

Twenty-seven control blood samples from healthy volunteers were collected as the control group. Exclusion criteria for the control group included cancer, infection, or 
any other immune-related disease that may influence the study.

Plasma samples were collected from blood samples in EDTA tubes after centrifugation $(3,000 \mathrm{r} / \mathrm{min}, 10 \mathrm{~min})$ and stored at $-80{ }^{\circ} \mathrm{C}$. PBMCs were isolated by FicollHypaque density gradient centrifugation according to the manufacturer's protocols (TBD, Tianjin, China). Collected PBMCs were suspended in radioimmunoprecipitation assay (RIPA) lysis buffer (Beyotime, Jiangsu, China) and stored at $-80{ }^{\circ} \mathrm{C}$.

\section{Purification and characterization of recombinant $K S$}

Recombinant human KS with a C-his Tag was obtained from Sino Biological Inc. (Beijing, China). Briefly, a pCMV3-SP-N-Myc-his-C plasmid encoding SERPINA4 was transiently transfected into human embryonic kidney cells (HEK293H). Recombinant human KS was expressed in cultured HEK293H cells, and cultured medium was concentrated by ammonium sulphate precipitation followed by immobilized metal affinity chromatography. Purified recombinant human $\mathrm{KS}$ was identified by Coomassie blue staining and western blot.

\section{AngII-infused AAA models and sample collection}

$\mathrm{ApoE}^{-/-}$mice on a C57BL/6 background were purchased from Vital River Laboratories (Beijing, China). All mice were housed in a pathogen-free barrier facility at $22 \pm 2{ }^{\circ} \mathrm{C}$ with a relative humidity of $55 \% \pm 5 \%$ and a 12 -h dark: light cycle. As previously reported (16), from week 10, mice were fed a standard commercial diet for 2 weeks, then maintained on high-fat diets (Keao Xieli Feed, Beijing, China) (48.6\% kcal from fat, $0.2 \%$ cholesterol) with water provided ad libitum for 4 weeks. Subsequently, male $\mathrm{ApoE}^{-/-}$mice were divided into the following 3 groups: (I) ten mice were infused with saline using mini-osmotic pumps (Model 2004, Alzet, DURECT Corporation, San Diego, CA, USA) for 28 days, as the Saline group; (II) ten mice were infused with AngII (1,000 ng/kg/min; MCE, Shanghai, China) for 28 days by mini-osmotic pumps as the AngII group; and (III) AngII + KS group. Ten mice were infused with AngII (1,000 $\mathrm{ng} / \mathrm{kg} / \mathrm{min})$ using mini-osmotic pumps for 28 days. Recombinant human KS $(0.5 \mathrm{mg} / \mathrm{kg} /$ day $)$ was administered daily through subcutaneous injection from day 7 to 28 after AngII infusion. Mice in these three groups were sacrificed on day 28 under pentobarbital anesthesia. Obtained aortic walls were divided into two parts and either stored at $-80{ }^{\circ} \mathrm{C}$ or fixed in $4 \%$ paraformaldehyde for paraffin sections.

\section{$R N A$ extraction and quantitative real-time PCR (qRT- PCR) analysis}

Total mRNA was extracted from aortic tissue samples using TRIzol reagent (Takara Bio, Shiga, Japan) as described previously (17). High-quality RNA samples had an A260/ A280 ratio of $>1.8$. qRT-PCR was performed using SYBR Premix Ex TaqII (RR820A; Takara Bio, Shiga, Japan), after synthesizing cDNA using PrimeScript RT reagent kits (RR037A; Takara Bio), with a modified amplification protocol: initial denaturation step at $95{ }^{\circ} \mathrm{C}$ for $30 \mathrm{~s}$, then 40 cycles of $95^{\circ} \mathrm{C}$ denaturation for $5 \mathrm{~s}$, and annealing and extension at $60{ }^{\circ} \mathrm{C}$ for $30 \mathrm{~s}$. RT-PCR analysis for all samples was independently repeated at least twice. All primers were purchased from Sangon (Shanghai, China): KS (SERPINA4), GAPDH, MSR-1, CD45, CD3, SMTN, MYH10, MYH11, $V C A M-1$, and Coll I, wnt3, beta-catenin (CTNNB1), $G S K 3 B, I C A M-1, V E G F A$, and TNFA, and their sequences are shown in Table $S 1$.

\section{Western blot analysis}

Protein samples were analyzed by sodium dodecyl sulfate polyacrylamide gel electrophoresis. After electrophoresis, the separated proteins were transferred onto polyvinylidene difluoride membranes, blocked with 5\% non-fat dry milk in Tris-buffered saline with $0.05 \%$ Tween 20 (pH 7.4) for $1 \mathrm{~h}$ at room temperature, followed by incubation with the primary antibody overnight at $4{ }^{\circ} \mathrm{C}$. The antibodies used in the study were: anti-KS (dilution 1:1,000; Abcam, Cambridge, UK), anti-LRP6 (dilution 1:500; Cell Signaling Technology, Beverly, MA, USA), anti-phospho-LRP6 (P-LRP6) (dilution 1:500; Cell Signaling Technology), antiGAPDH (dilution 1:2,000; Zsbio, Beijing, China), antiICAM-1 (dilution 1:500, Proteintech, Wuhan, China), and anti-TNF- $\alpha$ (dilution 1:500; Absin, Shanghai, China). After three washes, blots were incubated with the appropriate horseradish peroxidase-conjugated secondary antibodies (dilution 1:10,000, Zsbio) for $1 \mathrm{~h}$ at room temperature, and proteins were detected using BeyoECL Star (Beyotime, Beijing, China). Image J software 1.47 (Research Services Branch, National Institutes of Health, Bethesda, MD, USA) was used for protein analysis. GAPDH expression was used for normalization. 


\section{Histological analysis}

Representative sections of human and mouse aortic tissue samples $(2-3 \mu \mathrm{m})$ were used for staining. Paraffin sections were routinely stained with hematoxylin and eosin (HE) and Elastica van Gieson (EvG) to examine tissue morphology, cellular composition, the degree of infiltration by inflammatory cells, and the content of elastin and collagen fibers in AAA samples, according to standard protocols.

We characterized the elastin integrity of aortas in mice using EvG staining, which demonstrated extensive elastin degradation within the aortic wall, as previously described $(13,18)$. Semi-quantitative evaluation of elastin filament integrity was performed by a blinded observer on digital images, as previously described $(12,18)$. Briefly, they were graded as follows: 1 -no elastin filament degradation; 2mild elastin filament distension; 3-moderate-to-severe elastin filament degradation; and 4-severe elastin filament degradation.

\section{Immunobistochemical (IHC) staining analysis}

For IHC, paraffin sections were deparaffinized and rehydrated in gradient concentrations of ethanol. They were then boiled in EDTA acid or citrate buffer to retrieve antigen epitopes, washed with phosphate-buffered saline, and treated with the appropriate antibodies. Consecutive slides were prepared for co-localization analysis. One slide was stained with an antibody to detect the specific cell type and a consecutive slide was stained with an antibody against the individual biomarker factor of interest. Smooth muscle cells (SMCs) were detected by an anti-alpha-smooth muscle actin (anti- $\alpha$-SMA) antibody (dilution 1:1,000; Absin), endothelial cells (ECs) by an anti-CD34 antibody (dilution 1:1,000; Proteintech), white blood cells by an anti-CD45 antibody (dilution 1:500; Proteintech), macrophages by an anti-CD68 antibody (dilution 1:500; Proteintech), and T lymphocytes by an anti-CD3 antibody (dilution 1:400; Absin) according to the manufacturer's instructions. The quality of each slide was evaluated twice independently as follows: 0 -no staining; 1 -weak positive staining; $2-$ scattered positive staining; 3-majority of positive staining; and 4-strong overall positive staining.

\section{Enzyme-linked immunosorbent assay (ELISA)}

ELISA was performed for the quantitative determination of KS concentrations in plasma according to the manufacturer's protocols (Cusabio, Wuhan, China). The intensity of color was measured at $450 \mathrm{~nm}$. A standard curve was constructed and used to determine the KS concentration in plasma.

\section{Immunofluorescence (IF) staining of KS and a-SMA}

Representative paraffin sections $(2-3 \mu \mathrm{m})$ were deparaffinized and rehydrated in gradient concentrations of ethanol, boiled in EDTA acid or citrate buffer to retrieve antigen epitopes, washed with phosphate-buffered saline, and incubated with a mixture of rabbit anti-KS antibody (dilution 1:350; Abcam) and goat anti- $\alpha$-SMA antibody (dilution 1:400; Abcam) overnight at $4{ }^{\circ} \mathrm{C}$ in a humidified chamber. Sections were washed 3 times with Tris-buffered saline, incubated with the mixture of FITC coupled to donkey anti-rabbit antibody (dilution 1:200; Abcam) and TRITC coupled to donkey anti-goat antibody (dilution 1:200; Abcam) for 1 hour. The sections were acquired with a confocal microscope (Nikon CI plus, Tokyo, Japan).

\section{Chromogenic in situ hybridization (CISH)}

CISH was performed according to manufacturer instructions as described previously. All reagents and instruments were treated with diethylpyrocarbonate (DEPC) and the whole process was carried out in the DNase/RNase-free environment. In brief, aortic tissue samples were fixed in $4 \%$ paraformaldehyde for paraffin sections. tissue sections $(2-3 \mu \mathrm{m})$ were deparaffinized, rehydrated, and treated with $20 \mu \mathrm{g} / \mathrm{mL}$ of proteinase $\mathrm{K}$ (G1004, Servicebio, Wuhan, China) for 20 minutes at $37^{\circ} \mathrm{C}$. After blockaded peroxidase with $3 \% \mathrm{H} 2 \mathrm{O} 2$ for 15 minutes, the sections were covered with in situ hybridization solution (G3016-3, Servicebio) for 1 hour. Then hybridization was performed overnight in a humid chamber at $37{ }^{\circ} \mathrm{C}$ with a digoxin-labeled (DIG-labeled) SERPINA4 probe. After three washes, the sections were blocked by bovine serum albumin for 30 minutes. To detect the hybridization signal, the sections were incubated with a mouse anti-DIG-labeling antibody conjugated with horseradish peroxidase (200002-156, Jackson ImmunoResearch Inc., USA) at $37{ }^{\circ} \mathrm{C}$ for 40 minutes. The hybridization signal was detected by a diaminobenzidine substrate kit (G1211, Servicebio). After development, the slides were mounted with coverslips. The specific sequence of DIG-labeled SERPINA4 probe: 5'-DIG-GGTTGCGTCTCCTTTGTAATCCATCCGT AG-3'. 
Table 1 Demographic and clinical characteristics of AAA patients and controls included in this study

\begin{tabular}{|c|c|c|c|c|c|}
\hline Characteristics & $\begin{array}{l}\text { AAA group } \\
\quad(\mathrm{N}=36)\end{array}$ & \multicolumn{2}{|c|}{ Tissue samples } & \multicolumn{2}{|c|}{ Blood samples } \\
\hline Age, mean \pm SEM (year) & $64.69 \pm 1.05$ & $61.33 \pm 1.68$ & 0.127 & $64.22 \pm 0.76$ & 0.716 \\
\hline Female, $\mathrm{n}$ & 3 & 1 & 1.000 & 3 & 0.710 \\
\hline Maximum AAA diameter, mean \pm SEM $(\mathrm{mm})$ & $66.15 \pm 18.78$ & $18.93 \pm 0.86$ & $\mathrm{P}<0.001^{\mathrm{a}}$ & $19.22 \pm 0.99$ & $\mathrm{P}<0.001^{\mathrm{a}}$ \\
\hline $\mathrm{DM}$ & 10 & 2 & 0.441 & 3 & 0.106 \\
\hline Hypertension & 15 & 3 & 0.302 & 7 & 0.195 \\
\hline Smoking & 13 & 2 & 0.208 & 5 & 0.125 \\
\hline Carotid disease & 2 & 0 & 0.404 & 0 & 0.213 \\
\hline Hyperlipidemia & 7 & 1 & 0.371 & 2 & 0.177 \\
\hline
\end{tabular}

a, statistically significant. AAA, abdominal aortic aneurysm; DM, diabetes mellitus; SEM, standard error of the mean.

\section{Statistical analysis}

We used SPSS for Windows version 22.0 (SPSS Inc., Chicago, IL, USA) for statistical analysis. KolmogorovSmirnov's one sample nonparametric test was used to determine the normal distribution of variables. Continuous variables were compared either by the parametric $t$-test for unpaired samples or the non-parametric Mann-Whitney $\mathrm{U}$ test for comparisons between two groups. Comparisons among three groups were performed by one-way analysis of variance (ANOVA), followed by Tukey's post-hoc test, or nonparametric tests, followed by Kruskal-Wallis 1-way ANOVA test, according to the normality of the values. Correlations between continuous variables were analyzed by Pearson's correlation coefficient for normally distributed values or Spearman's rank correlation coefficient for nonparametric samples. Chi-square test was used to determine differences in categorical variables between groups. In addition, binary logistic regression analysis was performed to assess the independent associations between KS levels and cardiovascular risk factors. Data were presented as the mean \pm standard deviation (SD) for variables with a normal distribution or median (min, max) for variables without a normal distribution. $\mathrm{P}$ values $<0.05$ were considered statistically significant.

\section{Results}

\section{Characteristics of study subjects}

Demographic characteristics and prevalence of comorbidities of AAA patients and controls were summarized in Table 1. For the tissue samples included in this study, the two groups were similar in age and gender $(\mathrm{P}=0.127$, and $\mathrm{P}=1.000)$. And there were no significant differences between AAA patients and controls regarding the prevalence of comorbidities, including diabetes mellitus $(\mathrm{DM})$, hypertension, smoking history, carotid disease, cardiac disease, renal disease and hyperlipidemia $(\mathrm{P}=0.441$, $\mathrm{P}=0.302, \mathrm{P}=0.208 \mathrm{P}=0.404, \mathrm{P}=0.614, \mathrm{P}=0.614$, and $\mathrm{P}=0.371$ respectively) (Figure $S 1$ ). No obvious atherosclerotic changes within the aortic walls of control subjects were observed by light microscopy. For the blood samples included in this study, there were no significant differences in age and gender between the two groups $(\mathrm{P}=0.716$, and $\mathrm{P}=0.710$ respectively). Moreover, there were no significant differences between AAA patients and controls regarding the prevalence of comorbidities, including diabetes mellitus (DM), hypertension, history of smoking, carotid disease, cardiac disease, and renal disease and hypertension $(\mathrm{P}=0.106, \mathrm{P}=0.195, \mathrm{P}=0.125, \mathrm{P}=0.213, \mathrm{P}=0.418, \mathrm{P}=0.173$, and $\mathrm{P}=0.177$ respectively). AAA patients included in this 
study had a relative healthy peripheral vascular system as checked by CTA and no evidence or medical history of other autoimmune diseases. Individuals included in the control groups had no evidence or medical history of vascular disorders. Table 2 shows the symptoms and blood parameters and their corresponding cut-off value of AAA patients included in the present study.

The expression and cellular localization of $K S$ in $A A A$ tissues

KS expression at the protein level was significantly higher

Table 2 Clinical data of AAA patients

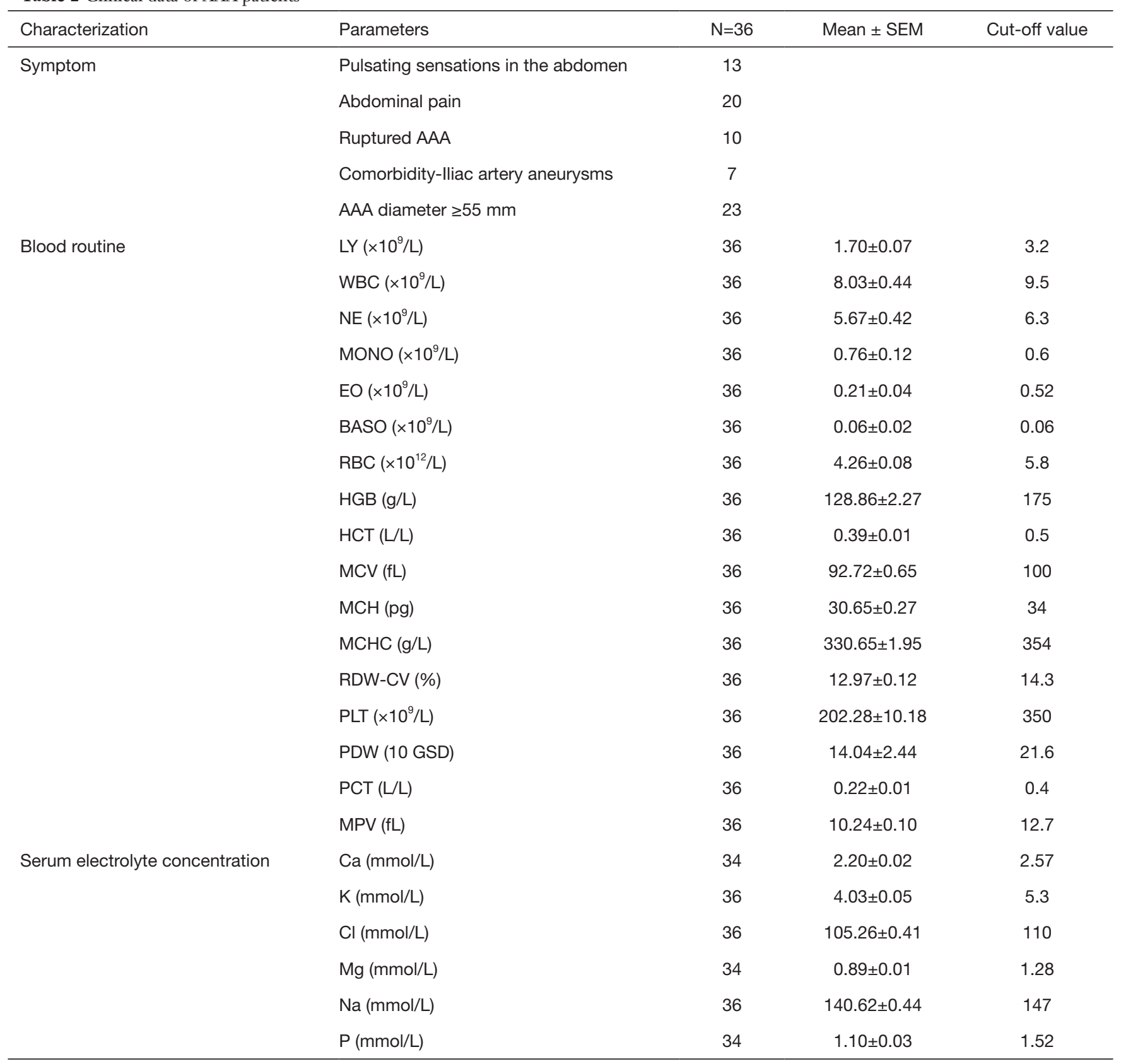

Table 2 (continued) 
Table 2 (continued)

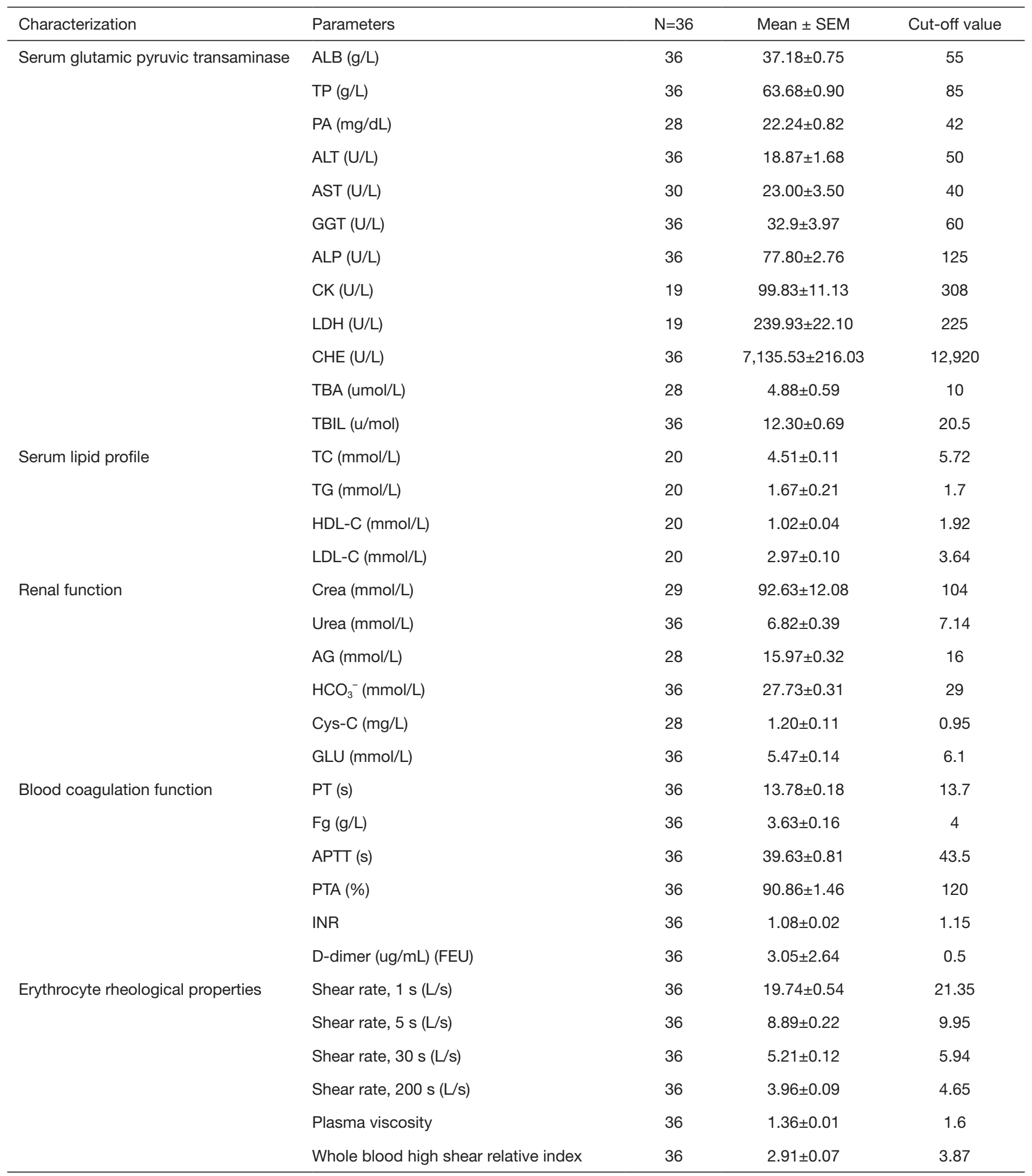

Table 2 (continued) 
Table 2 (continued)

\begin{tabular}{|c|c|c|c|c|}
\hline Characterization & Parameters & $\mathrm{N}=36$ & Mean \pm SEM & Cut-off value \\
\hline & Whole blood low shear relative viscosity & 36 & $39.68 \pm 0.50$ & 50.38 \\
\hline & Whole blood high shear relative viscosity & 36 & $5.59 \pm 0.12$ & 8.62 \\
\hline & Red cell aggregation index & 36 & $4.98 \pm 0.07$ & 6.05 \\
\hline & Red cell deformation index & 36 & $0.75 \pm 0.01$ & 1.05 \\
\hline & Hematocrit (L/L) & 36 & $0.46 \pm 0.01$ & 0.49 \\
\hline & $\mathrm{ESR}(\mathrm{mm} / \mathrm{h})$ & 36 & $22.30 \pm 3.24$ & 15 \\
\hline
\end{tabular}

AAA, abdominal aortic aneurysm; SEM, standard error of the mean; LY, lymphocyte; WBC, white blood cells; N, neutrophil; MONO, monocyte; EO, eosinophils; BASO, basophil; RBC, red blood cells; HGB, hemoglobin; HCT, hematocrit; MCV, mean corpuscular volume; $\mathrm{MCH}$, mean corpuscular hemoglobin; RDW-CV, red blood cell volume distribution width; MCHC, mean corpuscular hemoglobin concentration; PLT, platelets; PDW, platelet distribution width; PCT, platelet hematocrit; MPV, mean platelet volume; Ca, serum calcium; $\mathrm{K}$, serum kalium; CL, serum chlorine; Mg, serum magnesium; $\mathrm{Na}$, serum sodium; P, serum phosphate; ALB, albumin; TP, total protein; PA, pre-albumin; ALT, alanine aminotransferase; AST, aspartate aminotransferase; GGT, gamma glutamyl transpeptidase; ALP, alkaline phosphatase; CK, creatine kinase; LDH, lactate dehydrogenase; CHE, cholinesterase; TBA, total bile acid; TBIL, total bilirubin; TC, total cholesterol; TG, triglycerides; HDL-C, high-density lipoprotein-cholesterol; LDL-C, low-density lipoprotein-cholesterol; Crea, creatinine; AG, anion gap; Cys-C, cystatin C; GLU, fasting plasma glucose; PT, prothrombin time; APTT, activated partial thromboplastin time; PTA, prothrombin activity, INR, international normalized ratio; ESR, erythrocyte sedimentation rate.

in AAA tissue samples compared with control aortic samples $(\mathrm{P}<0.001$, Figure $1 A, B)$ as assessed by western blot analysis, which was also supported by IHC analysis $(\mathrm{P}<0.001)$. Representative images of these results were shown in Figure $1 C, D$.

Subsequently, we analyzed the cellular localization of KS within the AAA wall by IHC in consecutively stained sections. KS was strongly co-localized with SMCs. And there was predominantly granule-like KS staining in the media, adventitia and intramural. However, the staining patterns of KS were not observed to co-localized with CD $34^{+}$ECs, leucocytes, macrophages, and T cells (Figure $1 E$ ). To further investigate the expression of KS in SMCs of AAA samples, IF staining was performed to evaluate $\mathrm{KS}$ and $\alpha$-SMA. IF staining results also showed that KS was located in the SMCs (Figure S2).

In addition, we also detected the expression of SERPINA4 mRNA in AAA tissue samples using qRT-PCR. Compared with control aortic tissue samples, the expression of SERPINA4 was significantly decreased in AAA samples $(\mathrm{P}=0.018$, Figure $1 F)$. And the expression of SERPINA4 mRNA was observed located in the healthy aorta using CISH analysis (Figure S3).

We were unable to isolate individual cells from AAA tissue samples to confirm the location of SERPINA4; therefore, we correlated SERPINA4 mRNA expression with markers indicative of different cell types. Smoothelin $(S M T N)$ and smooth muscle myosin heavy chain (SM-MHC, MYH11) were selected to represent the contractile phenotype, while SMemb/non-muscle MHCB (MYH10) and collagen I (Coll I) were chosen to represent the synthetic phenotype. MSR1 was selected to represent macrophages, $C D 3$ represented $\mathrm{T}$ lymphocytes, and $C D 45$ represented leucocytes. We also analyzed the correlation between SERPINA4 and vascular cell adhesion molecule-1 (VCAM-1), which plays an important role in the development of AAA (19). In AAA group, significantly negative correlations were observed between SERPINA4 and CD45, CD3, Coll I, MYH10, SM-MHC, and VCAM-1 $(\mathrm{r}=-0.688,-0.605,-0.597$, $-0.582,-0.644$ and -0.750 , and $\mathrm{P}<0.001, \mathrm{P}<0.001, \mathrm{P}<0.001$, $\mathrm{P}=0.023, \mathrm{P}<0.001$ and $\mathrm{P}<0.001$, respectively) (Table 3). In control group, significantly negative correlations in control tissue between SERPINA4 and MYH1O, Coll I and $S M-M H C(\mathrm{r}=-0.657,-0.636$ and -0.867 , and $\mathrm{P}<0.001$, $\mathrm{P}=0.020$ and $\mathrm{P}=0.026$, respectively). However, significant correlations were not observed between SERPINA4 and $C D 45$ and $C D 3$. The correlations in AAA group were stronger than them in the control group. 
A

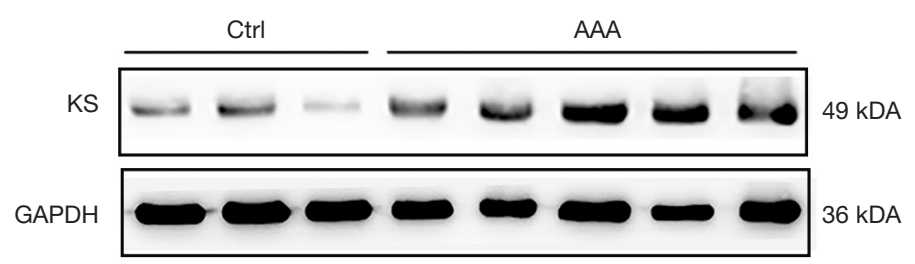

$\mathrm{C}$

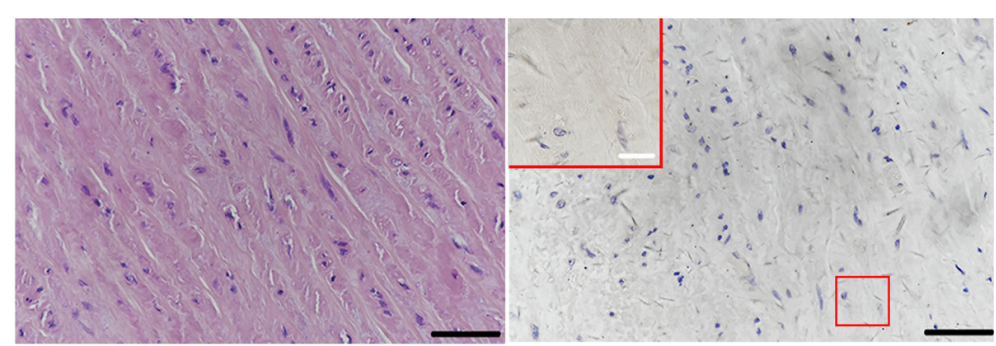

ii

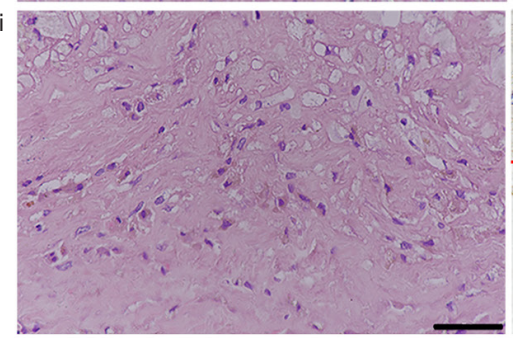

E

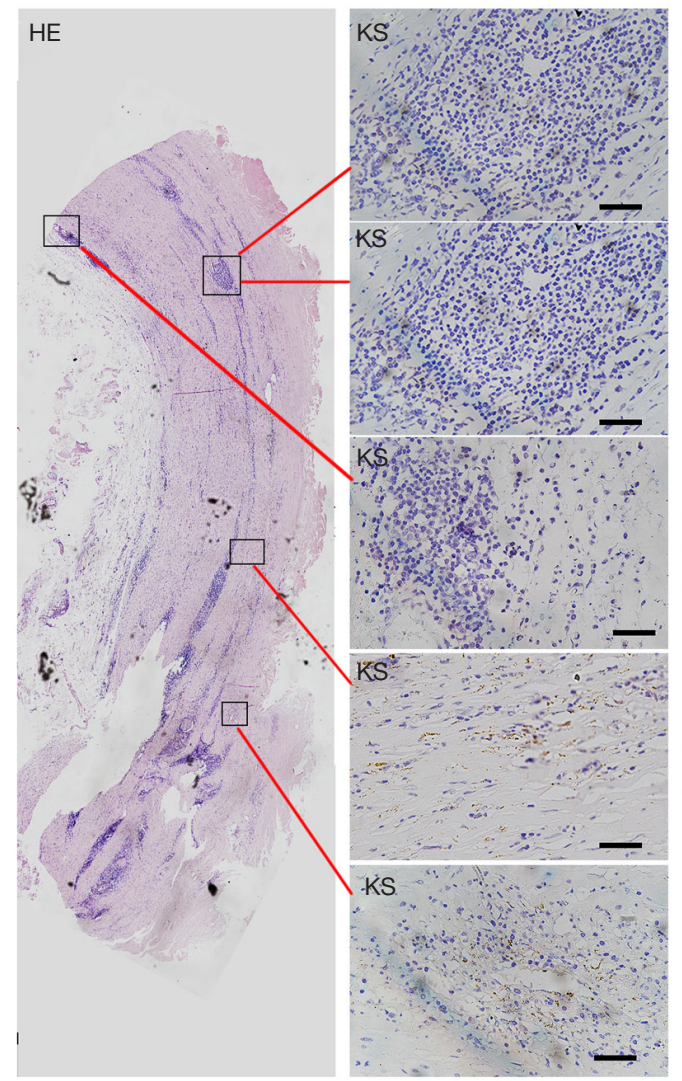

B

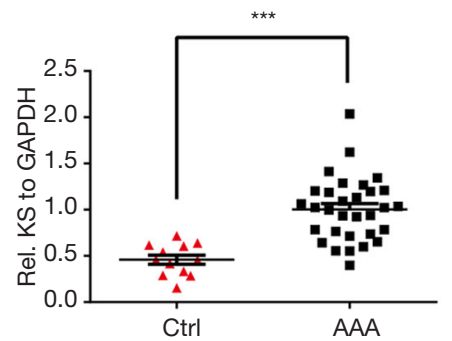

D

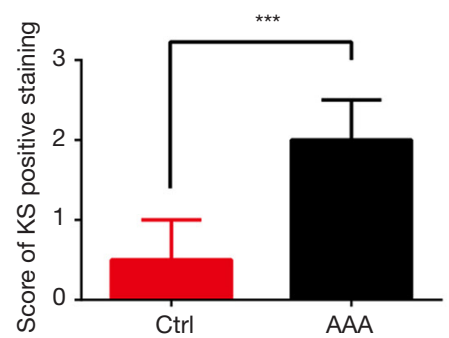

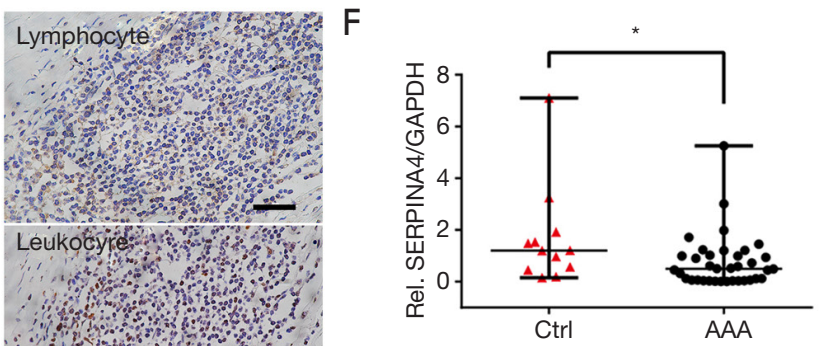


G

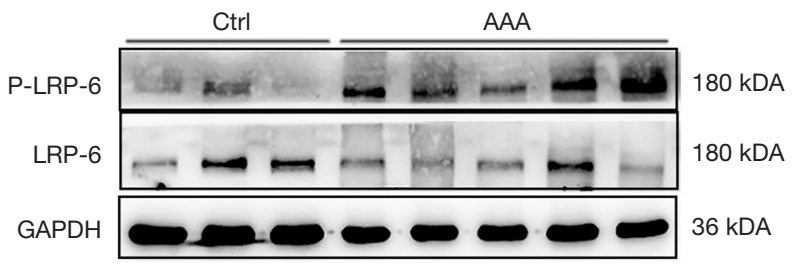

J

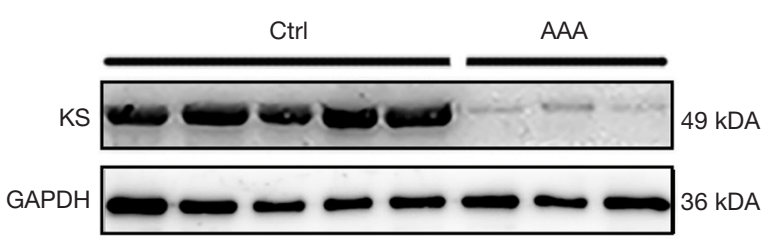

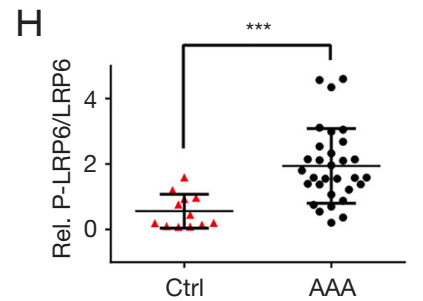

I

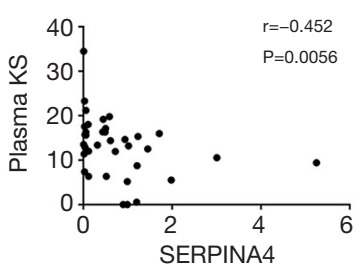

$\mathrm{K}$

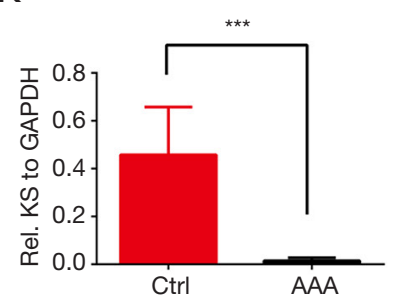

L

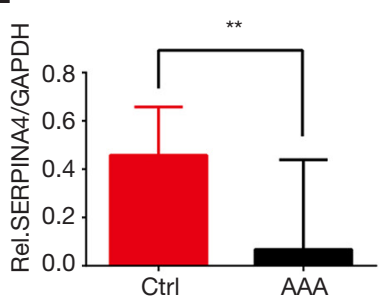

Figure 1 Proteins and genes expressions in AAA patients compared with the controls. (A) Representative Western blots of KS and (B) the expression analysis of KS in AAA and healthy aorta using the parametric $t$-test; (C) representative images of HE staining and KS staining in control healthy aortic tissues (i) and AAA (ii), and (D) the analysis of the score of KS positive staining by Mann-Whitney U test; (E) analysis of KS in AAA using IHC for cellular localization. Overview image (left panel) of the whole AAA tissue sample with areas selected for cellular localization of KS expression. The magnified images depict consecutive staining of individual cell types, as revealed by staining for indicated markers, within the AAA wall and indicated corresponding substrates; (F) SERPINA4 level in AAA tissue samples versus controls as examined by RT-PCR and analyzed by Mann-Whitney U test; $(\mathrm{G})$ representative western blots of total LRP6 and P-LRP6 and (H) the analysis of P-LRP6/total LRP6 in AAA and healthy aorta using the parametric $t$-test; (I) analysis of correlation between plasma KS levels and SERPINA4 mRNA expression in AAA patients; (J) representative western blots of KS and (K) the expression analysis of KS in PBMCs from AAA patients versus controls as examined by using Mann-Whitney U test; (L) SERPINA4 level in PBMCs from AAA patients versus controls as examined by RT-PCR and analyzed by Mann-Whitney $\mathrm{U}$ test. $\mathrm{P}$ values $<0.05$ were considered statistically significant: ${ }^{*}, \mathrm{P}<0.05$; **, $\mathrm{P}<0.01$; ***, $\mathrm{P}<0.001$. Scale bar, white: $10 \mu \mathrm{m}$; black: $50 \mu \mathrm{m}$. AAA, abdominal aortic aneurysm; KS, kallistatin; HE, hematoxylin and eosin; IHC, immunohistochemical; RT-PCR, real-time PCR; SMC, smooth muscle cell; EC, endothelial cell.

Table 3 Correlation between SERPINA4 and cell markers

\begin{tabular}{|c|c|c|c|c|c|c|c|c|}
\hline$r$ (SERPINA4) & $C D 45$ & $C D 3$ & MSR1 & SMTN & MYH10 & Coll I & $S M-M H C$ & VCAM-1 \\
\hline AAA group $(n=36)$ & $-0.688^{\star \star \star}$ & $-0.605^{\star * *}$ & 0.098 & -0.309 & $-0.582^{\star \star *}$ & $-0.597^{\star \star \star}$ & $-0.644^{\star \star \star}$ & $-0.750^{\text {*** }}$ \\
\hline Control group $(n=12)$ & -0.147 & 0.378 & 0.231 & -0.084 & $-0.657^{\star \star *}$ & $-0.636^{*}$ & $-0.867^{*}$ & -0.301 \\
\hline All $(n=48)$ & $-0.656^{\star \star \star}$ & $-0.480^{\star \star *}$ & 0.107 & -0.053 & $-0.327^{\star}$ & $-0.606^{\star \star *}$ & $-0.505^{\star \star \star}$ & $-0.641^{\star * *}$ \\
\hline
\end{tabular}

Significant differences between SERPINA4 and cell markers: ${ }^{*}, \mathrm{P}<0.05 ;{ }^{* * *}, \mathrm{P}<0.001$. AAA, abdominal aortic aneurysm.

\section{$K S$ is correlated with the canonical Wnt/ק-catenin pathway in buman $A A A$}

Previous studies (20) showed that KS (SERPINA4) blocked the canonical $W n t / \beta$-catenin pathway by competing with Wnt3 and Wnt3a to bind to LRP6. We therefore investigated the correlation between KS (SERPINA4) and the canonical $\mathrm{Wnt} / \beta$-catenin pathway in AAA. Wnt3
mRNA expression was significantly positively correlated with SERPINA4 expression in AAA $(\mathrm{r}=0.590, \mathrm{P}<0.001)$. Subsequently, significantly negative correlations with SERPINA4 expression were identified for the expression of Wnt pathway-related genes including CTNNB1, GSK3B, and Wnt pathway downstream genes including VEGFA and ICAM-1 $(\mathrm{r}=-0.664,-0.640,-0.560,-0.433$, and $\mathrm{P}<0.001$, 
Table 4 Correlation between SERPINA4 and Wnt signal pathway

\begin{tabular}{|c|c|c|c|c|c|c|c|}
\hline$r$ & SERPINA4 & GSK3B & CTNNB1 & WNT3 & TNFA & ICAM1 & VEGFA \\
\hline SERPINA4 & - & & & & & & \\
\hline GSK3B & $-0.640^{\star \star \star}$ & - & & & & & \\
\hline CTNNB1 & $-0.664^{\star \star \star}$ & $0.832^{\star \star *}$ & - & & & & \\
\hline WNT3 & $0.590^{\star \star \star}$ & $-0.313^{*}$ & NC & - & & & \\
\hline TNFA & NC & NC & $0.289^{*}$ & $0.648^{\star \star \star}$ & - & & \\
\hline ICAM1 & $-0.433^{\star \star}$ & $0.473^{\star \star}$ & $0.652^{\star \star \star}$ & NC & $0.721^{\star \star \star}$ & - & \\
\hline VEGFA & $-0.560^{\star \star \star}$ & $0.851^{\star \star \star}$ & $0.723^{\star \star \star}$ & $-0.333^{*}$ & NC & $0.541^{\star \star \star}$ & - \\
\hline
\end{tabular}

Significant differences between SERPINA4 and Wnt signal pathway: ${ }^{*}, \mathrm{P}<0.05 ;{ }^{* \star}, \mathrm{P}<0.01 ;{ }^{* \star *}, \mathrm{P}<0.001$. NC, no correlation.

$\mathrm{P}<0.001, \mathrm{P}<0.001, \mathrm{P}=0.002$, respectively) (Table 4).

Western blotting also showed that the ratio of P-LRP6 to total LRP6 was significantly upregulated $(\mathrm{P}=0.0002$, Figure $1 G, H)$ in AAA, representing activation of the Wnt signaling pathway at the receptor level. We observed that the relative quantity of KS was significantly positively correlated with the ratio of P-LRP6 to total LRP6 ( $\mathrm{r}=0.391$, and $\mathrm{P}=0.0095)$, which may indicate an immune reactive increase in KS.

\section{KS levels in blood samples from AAA patients and its correlation with clinical data}

We determined the plasma KS concentration by ELISA. Although there was no significant difference in plasma KS levels observed between two groups $(\mathrm{P}=0.11)$, plasma KS levels were negatively correlated with the matched mRNA expressions in the AAA group ( $\mathrm{r}=-0.452$, and $\mathrm{P}=0.0056$; Figure 1I).

A lower expression of $\mathrm{KS}$ in PBMCs was noted in AAA compared with controls $(\mathrm{P}<0.001$, Figure 1f,K). Similarly, SERPINA4 expression in PBMCs extracted from AAA patients was significantly downregulated compared with the control group by qPCR analysis $(\mathrm{P}=0.0015$, Figure $1 L)$.

\section{The association of tissue SERPINA4 mRNA level and plasma KS levels with human $A A A$ and their combined effects on $A A A$}

Although there was no significant difference in plasma KS levels between two groups, Spearman correlation analysis showed a significantly negative correlation between SERPINA4 mRNA levels and matched plasma KS levels in the AAA group.

Subsequently, we analyzed the correlations between
SERPINA4 mRNA levels and clinical blood parameters. In routine blood examination, SERPINA4 levels were significantly correlated with lymphocyte count, monocyte count, monocyte ratio, red blood cell count, hemoglobin and platelet count $(\mathrm{r}=-0.383,-0.344,-0.395,-0.396,-0.338$ and -0.339 , and $\mathrm{P}=0.021, \mathrm{P}=0.040, \mathrm{P}=0.017, \mathrm{P}=0.017$, $\mathrm{P}=0.044$ and $\mathrm{P}=0.043$, respectively). SERPINA4 were also significant correlated with $\mathrm{LDH}$ and $\mathrm{D}$-dimer ( $\mathrm{r}=$ 0.520 and 0.366 , and $\mathrm{P}=0.022$ and $\mathrm{P}=0.028$, respectively). Furthermore, plasma KS levels were significantly correlated with hemoglobin and $\mathrm{D}$-dimer $(\mathrm{r}=0.340$ and -0.343 , and $\mathrm{P}=0.042$ and $\mathrm{P}=0.041$, respectively).

The adjusted logistic regression analysis was performed to show the effects of SERPINA4 mRNA, plasma KS and their combination on the AAA rupture, AAA diameter and other symptoms or signs (Table 5). As shown in Table 5, SERPINA4 levels were significantly negatively associated with the comorbidity of iliac artery aneurysm [odds ratio (OR): 0.017 ; 95\% confidence interval (CI): 0.001, $0.835 ; \mathrm{P}=0.040]$. Additionally, lower levels of plasma KS represented a higher risk of AAA rupture (OR: 0.837; 95\% CI: $0.710,0.987 ; \mathrm{P}=0.034)$. Subsequently, we evaluated the combined effects of SERPINA4 and plasma KS on AAA. According to our results, the combination of SERPINA4 mRNA and plasma KS was negatively associated with AAA rupture (OR: 0.661; 95\% CI: 0.472, 0.926; $\mathrm{P}=0.016$ ).

\section{Recombinant $K S$ results in attenuated aneurysm formation and inbibits the Wnt signaling patbway in AngII-infused ApoE ${ }^{--}$mice}

Purified recombinant human KS was identified by Coomassie blue staining and western blot (Figure S4). Although AngII infusion for 28 days in $\mathrm{ApoE}^{-/-}$mice 
Table 5 The adjusted logistic regression analysis of SERPINA4 expression, plasma KS and their combination at baseline and association with AAA clinical symptoms or signs

\begin{tabular}{|c|c|c|c|c|c|c|c|c|c|}
\hline Symptoms or signs & \multicolumn{3}{|c|}{ Model 1} & \multicolumn{3}{|c|}{ Model 2} & \multicolumn{3}{|c|}{ Model 3} \\
\hline \multicolumn{10}{|l|}{ SERPINA4 } \\
\hline Ruptured AAA & -1.178 & $0.308(0.068,1.479)$ & 0.144 & -1.228 & $0.293(0.058,1.479)$ & 0.137 & -1.203 & $0.300(0.053,1.713)$ & 0.176 \\
\hline Abdominal pain & -0.546 & $0.579(0.248,1.352)$ & 0.207 & -0.472 & $0.624(0.266,1.466)$ & 0.279 & -0.641 & $0.527(0.148,1.874)$ & 0.322 \\
\hline $\begin{array}{l}\text { Comorbidity-Iliac artery } \\
\text { aneurysms }\end{array}$ & -2.955 & $0.052(0.002,1.206)$ & 0.065 & -2.909 & $0.055(0.002,1.253)$ & 0.069 & -4.057 & $0.017(0.001,0.835)$ & 0.040 \\
\hline AAA diameter $\geq 55 \mathrm{~mm}$ & 0.183 & $1.201(0.566,2.548)$ & 0.633 & 0.313 & $1.367(0.571,3.273)$ & 0.483 & 0.430 & $1.537(0.578,4.090)$ & 0.389 \\
\hline \multicolumn{10}{|l|}{ Plasma KS } \\
\hline $\begin{array}{l}\text { Pulsating sensations in } \\
\text { the abdomen }\end{array}$ & -0.002 & $0.998(0.902,1.105)$ & 0.972 & -0.004 & $0.996(0.900,1.103)$ & 0.946 & 0.004 & $1.004(0.898,1.121)$ & 0.950 \\
\hline $\begin{array}{l}\text { Comorbidity-Iliac artery } \\
\text { aneurysms }\end{array}$ & 0.127 & $1.136(0.980,1.317)$ & 0.092 & 0.139 & $1.149(0.986,1.340)$ & 0.075 & 0.210 & $1.233(0.996,1.527)$ & 0.054 \\
\hline AAA diameter $\geq 55 \mathrm{~mm}$ & -0.093 & $0.911(0.809,1.027)$ & 0.126 & -0.092 & $0.912(0.808,1.029)$ & 0.134 & -0.108 & $0.898(0.787,1.038)$ & 0.111 \\
\hline \multicolumn{10}{|l|}{ Combination } \\
\hline Ruptured AAA & -0.247 & $0.781(0.649,0.940)$ & 0.009 & -0.313 & $0.731(0.518,0.925)$ & 0.009 & -0.413 & $0.661(0.472,0.926)$ & 0.016 \\
\hline
\end{tabular}

Model 1: no adjustments; model 2: adjusted for age, gender; model 3: additionally adjusted for hypertension, diabetes mellitus, hyperlipidemia and smoking on the base of model 2. KS, kallistatin; AAA, abdominal aortic aneurysm; $\beta$, regression coefficient; OR, odds ratio; $\mathrm{Cl}$, confidence interval.

contributed to AAA formation, the phenotype did not show complete penetration. The representative pictures of the mice in three groups were shown in Figure $2 A$. In our study, 6 mice in the AngII group $(\mathrm{n}=10)$ developed AAA at day 28 visibly, while 1 mouse developed AAA after KS infusion (Figure 2B). Approximately 20\% (2/10) of AngIIinfused aged mice died from aortic rupture, one at day 21 and one at day 26 respectively, while all mice in saline group and AngII + KS group had survived at day $28(\mathrm{P}=0.12)$. Additionally, AngII infusion caused a greater increase in maximal abdominal aortic diameter $(\mathrm{P}=0.001$; Figure $2 C)$, while compared with the AngII group, KS infusion decreased maximal abdominal aortic diameter $(\mathrm{P}=0.045)$.

Histological characteristics, including tissue morphology, cellular composition and inflammatory cell infiltration, were evaluated by HE staining (Figure 2D). Semiquantitative analysis of CD45 IHC statining was used to reflect the inflammatory cell infiltration in aortic walls in the control group, the AngII group and the AngII + KS group (Figure S5). EvG staining was performed to evaluate the elastin integrity in the three groups. Semi-quantitative measurements of elastin degradation showed significantly increased elastin degradation in the AngII group compared with mice in the saline group $(\mathrm{P}<0.001$, Figure $2 D, E, F)$. After KS infusion, elastin degradation was decreased $(\mathrm{P}=0.037)$.

Western blotting showed that P-LRP6 and betacatenin were significantly increased in the AngII groups compared with the saline groups (Figure $2 G, H, I$ ), $\mathrm{P}=0.004$ and $\mathrm{P}<0.001$, respectively). By contrast, $\mathrm{KS}$ treatment resulted in a significant decrease in P-LRP6 $(\mathrm{P}=0.013)$ and beta-catenin levels $(\mathrm{P}<0.001)$. Similarly, ICAM-1 protein levels were significantly increased after AngII infusion $(\mathrm{P}=0.008$, respectively; Figure $2 \mathrm{f}, \mathrm{K})$, as measured by western immunoblot. Its levels in the AngII + KS groups were lower than in the AngII groups $(\mathrm{P}=0.045)$. 
A
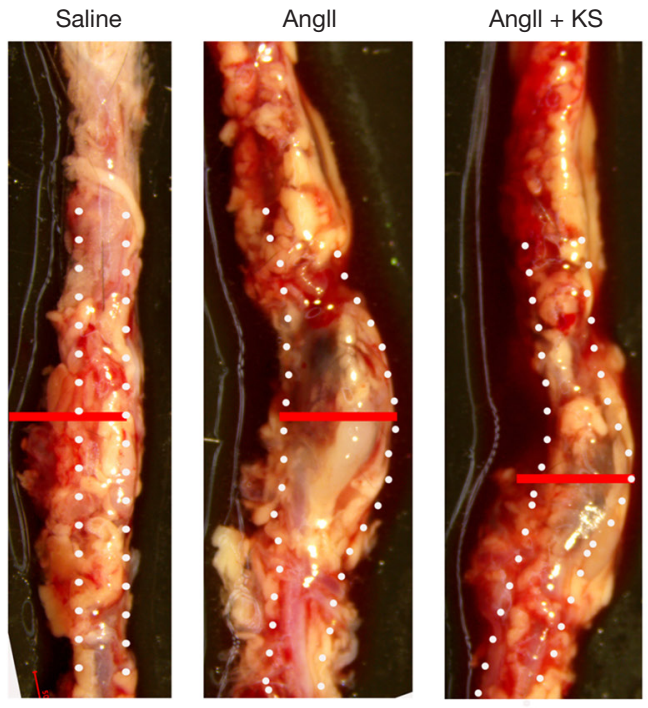

D

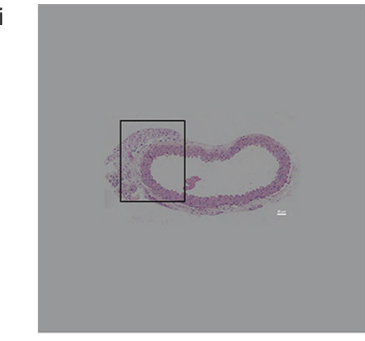

ii

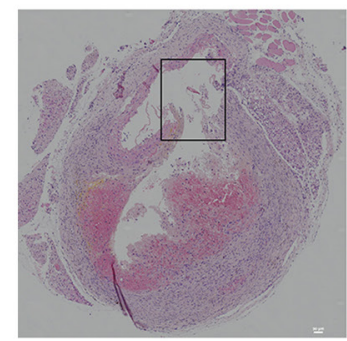

iii

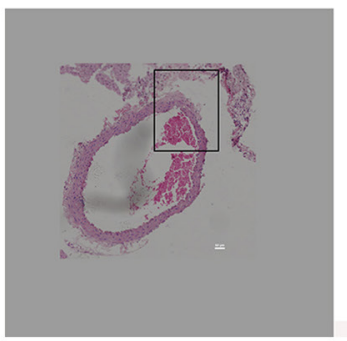

Angll + KS

C

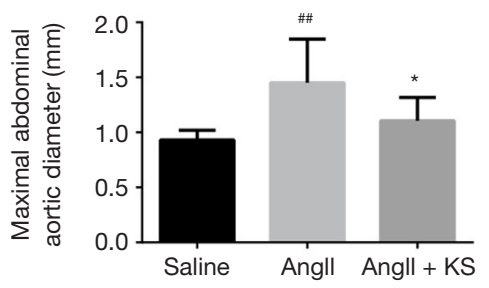

E

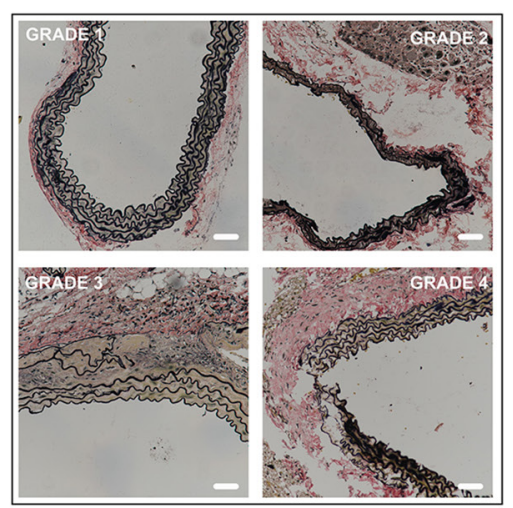

$\mathrm{F}$

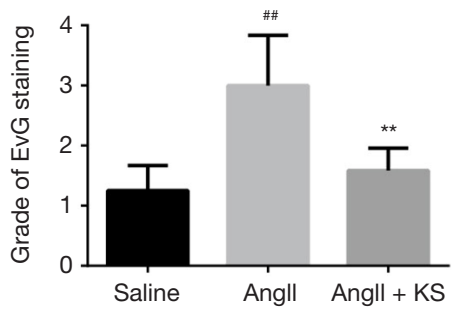


G

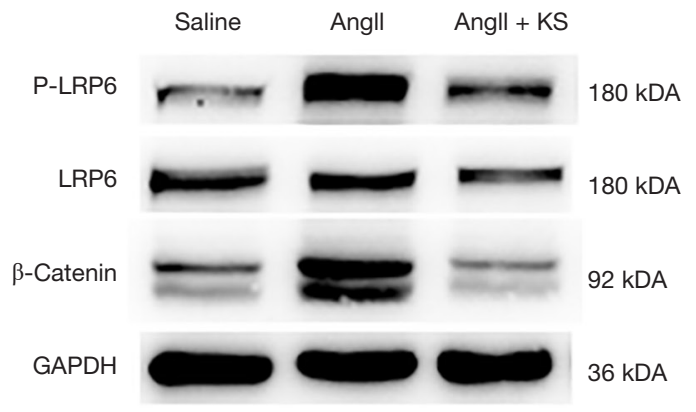

J
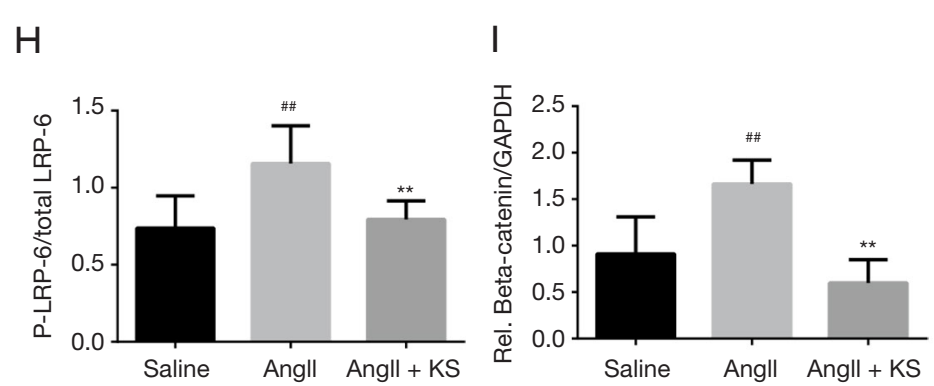

$\mathrm{K}$
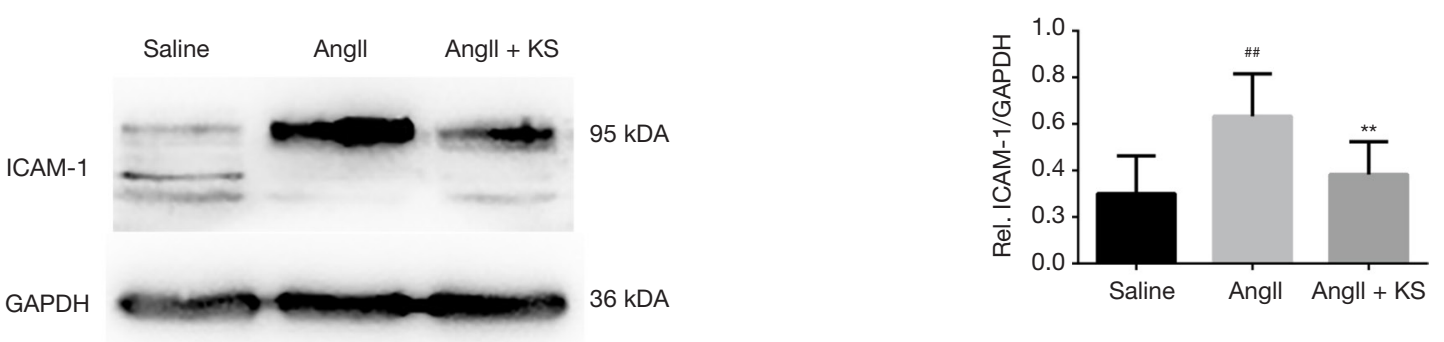

Figure 2 The evaluation of AngII infused $\mathrm{ApoE}^{-1-} \mathrm{AAA}$ model and the role of recombinant $\mathrm{KS}$ in the AAA model. (A) The representative images of aorta in the three groups; (B) the analysis of the incidence of AAA and (C) the analysis of maximal abdominal aortic diameters among groups; (D) HE-stained section and the EvG-stained sections of suprarenal aortic sections from (i) Saline group, (ii) AngII group and (iii) AngII + KS group; (E) semi-quantitative evaluation of elastin filament integrity in aorta tissue sample. Elastin flament degradation was graded on a scale of 1 to 4; (F) the grade of elastin filament integrity in aorta tissue sample from the saline group, AngII group and AngII + KS group; $(G)$ representative western blots of relative proteins in Wnt signal pathway and the expression analysis of $(\mathrm{H})$ the ratio of P-LRP6/total LRP6 and (I) beta-catenin in the saline group, AngII group and AngII + KS group; (J) representative Western blots of ICAM1, normalized by GAPDH, and (K) the expression analysis of ICAM-1 in the saline group, AngII group and AngII + KS group. Scale bar, $50 \mu \mathrm{m} .{ }^{\text {\#\# }}, \mathrm{P}<0.01 v s$. the saline groupand ${ }^{*}, \mathrm{P}<0.05,{ }^{* *}, \mathrm{P}<0.01 v s$. the AngII group. Data are presented as means $\pm \mathrm{SEM}$. AngII, angiotensin II; $\mathrm{ApoE}^{---}$, apolipoprotein E-deficient; KS, kallistatin; AAA, abdominal aortic aneurysm; HE, hematoxylin and eosin; EvG, Elastica van Gieson; SEM, standard error of the mean.

\section{Discussion}

Many studies have reported that KS has direct vascular effects and plays a crucial protective role in some cardiovascular diseases (11,21-25). However, studies on the involvement between KS and aortic diseases are lacking. For the first time, the current study demonstrates a significant difference in KS levels between AAA patients and controls, indicating the potential role of KS in the pathogenesis of AAA.

According to our results, SERPINA4 mRNA expression was downregulated in AAA tissues compared with controls, and SERPINA4 expression was significantly negatively correlated with the expression of inflammation-related genes, including $C D 3, C D 45, I C A M-1$, and VEGFA. CD3 and $C D 45$ are cell markers representing $\mathrm{T}$ lymphocytes and leukocytes, whereas ICAM-1 and VEGFA are critical pro-inflammatory and pro-angiogenesis mediators in vascular inflammation (26), and are also associated with the Wnt signaling pathway (6). The anti-inflammatory role of SERPINA4 has been widely recognized in different pathogeneses. In some inflammatory processes such as a Group A Streptococcus-infected mouse model, overexpressed SERPINA4 enhanced mouse survival and suppressed the viability of infiltrating cells, including neutrophils and $\mathrm{T}$ lymphocytes (27). Other studies have shown that the administration of KS suppresses the expression of many pro-inflammatory mediators including 
VEGFA and ICAM-1 $(6,14)$. Combining the antiinflammatory role of KS reported in previous studies and the inflammatory mechanism of AAA, our results indicated that the decreased expression of SERPINA4 in inflammatory cells may promote an inflammatory reaction in AAA, including the infiltration and accumulation of inflammatory cells and the progression of AAA. Previous in vitro and in vivo experiments have shown that KS inhibits inflammation and angiogenesis by blocking the Wnt signaling pathway (6). In this study, we found that SERPINA4 was negatively correlated with the expression of genes associated with $\mathrm{Wnt}$ signaling. The results indicated that KS may be a potential protective factor in aortic walls.

Interestingly, we observed that KS protein levels were up-regulated in AAA tissue samples, which was inconsistent with SERPINA4 expression. According to IHC analysis, we observed that KS was co-localized with SMCs, and there was predominantly granule-like KS staining in the media and adventitia. As previously reported $(28,29)$, KS is mainly produced by the liver, and present at high levels in plasma, and can be detected in blood vessels. In addition, it has been previously reported (30) that subcutaneous exogenous KS protein administration could be detected in the lung tissues and prevents bleomycin-induced injury to lung structures in a rat model. Furthermore, KS is increasingly recognized as a valuable indicator for the diagnosis and evaluation of diseases, including diabetes (31), liver cirrhosis (32) and community-acquired pneumonia (33), and can cause a systemic inflammatory reaction as a selfprotective mechanism. Therefore, we suggested the following hypothesis: KS (SERPINA4) in AAA walls decreased with the progression of AAA. Meanwhile, plasma KS might enter the aneurysmal walls and contribute to the upregulation of KS in AAA tissues as a self-protective mechanism. However, immune-reactive KS only plays a limited protective role, and is unable to effectively prevent AAA progression. This may partly explain the inconsistencies observed between KS expression at the mRNA and protein levels.

In the current study, although there was no significant differences in plasma KS levels between AAA patients and controls, upregulation of plasma KS represented a lower risk of rupture, which suggested its potential protective role in AAA. The combination of plasma KS levels and tissue SERPINA4 mRNA levels also showed significant negative association with AAA rupture with a lower OR value compared with the OR value of plasma KS alone. Human AAAs are usually asymptomatic until rupture, which is responsible for approximately half of the deaths related to AAA. Therefore, our results suggest that KS (SERPINA4) may be a protective factor against AAA by preventing rupture.

Clinically, surgical repair of AAA is the only available treatment, which includes open surgical repair (OSR) or endovascular aortic repair (EVAR) of the dilated aorta or ruptured AAA (34). However, surgical repair of AAA carries certain surgical risk of mortality and morbidity and late complications. Therefore, increased studies have focused on the medical therapy of AAA. For example, betablockers and angiotensin-converting enzyme inhibitors have been proved to be beneficial to reducing AAA growth, hypothetically by reducing either the wall shear stress or the inflammation (34). KS has the directive anti-inflammatory effects in cancer, sepsis and other diseases in several preclinical animal experiments (6). Additionally, according to our results, KS showed a potential protective role in AAA patients. Therefore, we established AngII-infused AAA mouse models to detect its potential therapeutic effects in AAA. In our study, we found that KS infusion reduced the incidence of AAA formation and enhanced the survival rate in AngII-infused $\mathrm{ApoE}^{-/-}$mice. In addition, the expression of beta-catenin, ICAM-1, and P-LRP6 were decreased in AngII-infused $\mathrm{ApoE}^{-/-}$mice after $\mathrm{KS}$ infusion. KS showed its protective roles in AAA mouse models, which indicates that KS might be a new target of drug therapy for AAAs.

Limitations in the current work should be considered. The study comprised a relatively small sample size. Additionally, we had to detect the cellular localizations of KS in formalin fixed human AAA tissue samples using IHC analysis of consecutively stained sections because we were unable to extract individual cells from AAA tissue samples. Moreover, as no appropriate antibody against KS was available for IHC analysis in AngII-infused $\mathrm{ApoE}^{--}$ mice, the detection of KS in histological structures in the AAA mouse model had to be omitted. Furthermore, in this study, we only observed that KS played a protective role in AAA. However, considering its diverse biological functions, further researches should focus on its mechanisms on preventing AAA growth.

\section{Conclusions}

In this study, significant difference of KS (SERPINA4) expression were observed in AAA, compared with the controls. Our findings also indicate that KS plays an important anti-inflammatory role and shows strong 
clinical correlations in AAA. Additionally, decreased KS (SERPINA4) level is a risk factor of AAA rupture. Furthermore, the pre-clinical animal experiments demonstrate that treatment with recombination KS suppresses AngII-induced aortic inflammation. This study suggests the potential therapeutic benefit of KS therapy in preventing aortic aneurysm.

\section{Acknowledgment}

We thank Edanz Group China (www.liwenbianji.cn/ac), for editing the English text of a draft of this manuscript. Funding: This work was supported by basic research program of Liaoning provincial institutions of higher learning 2017(LZDK201701), National Natural Science Foundation of China (grant number: 81970402), and another National Natural Science Foundation of China (grant number: 81600370).

\section{Footnote}

Conflicts of Interest: All authors have completed the ICMJE uniform disclosure form (available at http://dx.doi. org/10.21037/cdt.2019.12.08). The authors have no conflicts of interest to declare.

Ethical Statement: The authors are accountable for all aspects of the work in ensuring that questions related to the accuracy or integrity of any part of the work are appropriately investigated and resolved. Participants gave written informed consent before the study, and the study protocol was approved by the Ethics Committee of the First Hospital of China Medical University (No. AF-SOP-07-1.1-01), in accordance with the Declaration of Helsinki.

Open Access Statement: This is an Open Access article distributed in accordance with the Creative Commons Attribution-NonCommercial-NoDerivs 4.0 International License (CC BY-NC-ND 4.0), which permits the noncommercial replication and distribution of the article with the strict proviso that no changes or edits are made and the original work is properly cited (including links to both the formal publication through the relevant DOI and the license). See: https://creativecommons.org/licenses/by-nc-nd/4.0/.

\section{References}

1. Sakalihasan N, Limet R, Defawe OD. Abdominal aortic aneurysm. Lancet 2005;365:1577-89.

2. Golledge J, Muller J, Daugherty A, et al. Abdominal aortic aneurysm: pathogenesis and implications for management. Arterioscler Thromb Vasc Biol 2006;26:2605-13.

3. Hirsch AT, Haskal ZJ, Hertzer NR, et al. ACC/AHA 2005 Practice Guidelines for the management of patients with peripheral arterial disease (lower extremity, renal, mesenteric, and abdominal aortic): a collaborative report from the American Association for Vascular Surgery/ Society for Vascular Surgery, Society for Cardiovascular Angiography and Interventions, Society for Vascular Medicine and Biology, Society of Interventional Radiology, and the ACC/AHA Task Force on Practice Guidelines (Writing Committee to Develop Guidelines for the Management of Patients With Peripheral Arterial Disease): endorsed by the American Association of Cardiovascular and Pulmonary Rehabilitation; National Heart, Lung, and Blood Institute; Society for Vascular Nursing; TransAtlantic Inter-Society Consensus; and Vascular Disease Foundation. Circulation 2006;113:e463-654.

4. Jalalzadeh $\mathrm{H}$, Indrakusuma R, Planken RN, et al. Inflammation as a predictor of abdominal aortic aneurysm growth and rupture: a systematic review of imaging biomarkers. Eur J Vasc Endovasc Surg 2016;52:333-42.

5. Zhou GX, Chao L, Chao J. Kallistatin: a novel human tissue kallikrein inhibitor. Purification, characterization, and reactive center sequence. J Biol Chem 1992;267:25873-80.

6. Li J, Krishna SM, Golledge J. The potential role of kallistatin in the development of abdominal aortic aneurysm. Int J Mol Sci 2016. doi: 10.3390/ijms17081312.

7. Chao J, Li P, Chao L. Kallistatin: double-edged role in angiogenesis, apoptosis and oxidative stress. Biol Chem 2017;398:1309-17.

8. Huang KF, Huang XP, Xiao GQ, et al. Kallistatin, a novel anti-angiogenesis agent, inhibits angiogenesis via inhibition of the NF- $\mathrm{\kappa B}$ signaling pathway. Biomed Pharmacother 2014;68:455-61.

9. Miao RQ, Murakami H, Song Q, et al. Kallistatin stimulates vascular smooth muscle cell proliferation and migration in vitro and neointima formation in ballooninjured rat artery. Circ Res 2000;86:418-24.

10. Guo Y, Li P, Gao L, et al. Kallistatin reduces vascular senescence and aging by regulating microRNA-34a-SIRT1 pathway. Aging Cell 2017;16:837-46.

11. Yin H, Gao L, Shen B, et al. Kallistatin inhibits vascular inflammation by antagonizing tumor necrosis factor-alphainduced nuclear factor kappaB activation. Hypertension 
2010;56:260-7.

12. Krishna SM, Seto SW, Jose RJ, et al. Wnt signaling pathway inhibitor sclerostin inhibits angiotensin IIinduced aortic aneurysm and atherosclerosis. Arterioscler Thromb Vasc Biol 2017;37:553-66.

13. Gay A, Towler DA. Wnt signaling in cardiovascular disease: opportunities and challenges. Curr Opin Lipidol 2017;28:387-96.

14. Liu X, Zhang B, McBride JD, et al. Antiangiogenic and antineuroinflammatory effects of kallistatin through interactions with the canonical Wnt pathway. Diabetes 2013;62:4228-38.

15. Zhang J, Yang Z, Li P, et al. Kallistatin antagonizes Wnt/ $\beta$-catenin signaling and cancer cell motility via binding to low-density lipoprotein receptor-related protein $6 . \mathrm{Mol}$ Cell Biochem 2013;379:295-301.

16. Daugherty A, Manning MW, Cassis LA. Angiotensin II promotes atherosclerotic lesions and aneurysms in apolipoprotein E-deficient mice. J Clin Invest 2000;105:1605-12.

17. Han Y, Tanios F, Reeps C, et al. Histone acetylation and histone acetyltransferases show significant alterations in human abdominal aortic aneurysm. Clin Epigenetics 2016;8:3.

18. Krishna SM, Seto SW, Jose RJ, et al. A peptide antagonist of thrombospondin-1 promotes abdominal aortic aneurysm progression in the angiotensin II-infused apolipoprotein-E-deficient mouse. Arterioscler Thromb Vasc Biol 2015;35:389-98.

19. Fukami K, Yamagishi S, Okuda S. Role of AGEs-RAGE system in cardiovascular disease. Curr Pharm Des 2014;20:2395-402.

20. Zhao L, Patel SH, Pei J, et al. Antagonizing Wnt pathway in diabetic retinopathy. Diabetes 2013;62:3993-5.

21. Gao L, Yin H, S Smith R Jr, et al. Role of kallistatin in prevention of cardiac remodeling after chronic myocardial infarction. Lab Invest 2008;88:1157-66.

22. Chao J, Bledsoe G, Chao L. Protective role of kallistatin in vascular and organ injury. Hypertension 2016;68:533-41.

23. Gao L, Li P, Zhang J, et al. Novel role of kallistatin in vascular repair by promoting mobility, viability, and function of endothelial progenitor cells. J Am Heart Assoc 2014;3:e01194.

24. Shen B, Smith RS, Hsu YT, et al. Kruppel-like factor 4 is a novel mediator of Kallistatin in inhibiting endothelial inflammation via increased endothelial nitric-oxide synthase expression. J Biol Chem 2009;284:35471-8.
25. Carlson TH, Kolman MR, Piepkorn M. Activation of antithrombin III isoforms by heparan sulphate glycosaminoglycans and other sulphated polysaccharides. Blood Coagul Fibrinolysis 1995;6:474-80.

26. Kaneko H, Anzai T, Takahashi T, et al. Role of vascular endothelial growth factor-A in development of abdominal aortic aneurysm. Cardiovasc Res 2011;91:358-67.

27. Lu SL, Tsai CY, Luo YH, et al. Kallistatin modulates immune cells and confers anti-inflammatory response to protect mice from group A streptococcal infection. Antimicrob Agents Chemother 2013;57:5366-72.

28. Chao J, Schmaier A, Chen LM, et al. Kallistatin, a novel human tissue kallikrein inhibitor: levels in body fluids, blood cells, and tissues in health and disease. J Lab Clin Med 1996;127:612-20.

29. Chai KX, Chen LM, Chao J, et al. Kallistatin: a novel human serine proteinase inhibitor. Molecular cloning, tissue distribution, and expression in Escherichia coli. J Biol Chem 1993;268:24498-505.

30. Huang $X$, Wang $X$, Xie X, et al. Kallistatin protects against bleomycin-induced idiopathic pulmonary fibrosis by inhibiting angiogenesis and inflammation. Am J Transl Res 2017;9:999-1011.

31. El-Asrar MA, Andrawes NG, Ismail EA, et al. Kallistatin as a marker of microvascular complications in children and adolescents with type 1 diabetes mellitus: Relation to carotid intima media thickness. Vasc Med 2015;20:509-17.

32. Cheng Z, Lv Y, Pang S, et al. Kallistatin, a new and reliable biomarker for the diagnosis of liver cirrhosis. Acta Pharm Sin B 2015;5:194-200.

33. Lin WC, Lu SL, Lin CF, et al. Plasma kallistatin levels in patients with severe community-acquired pneumonia. Crit Care 2013;17:R27.

34. Erbel R, Aboyans V, Boileau C, et al. 2014 ESC Guidelines on the diagnosis and treatment of aortic diseases: document covering acute and chronic aortic diseases of the thoracic and abdominal aorta of the adult. The Task Force for the Diagnosis and Treatment of Aortic Diseases of the European Society of Cardiology (ESC). Eur Heart J 2014;35:2873-926.

Cite this article as: He Y, Han Y, Xing J, Zhai X, Wang S, Xin S, Zhang J. Kallistatin correlates with inflammation in abdominal aortic aneurysm and suppresses its formation in mice. Cardiovasc Diagn Ther 2020;10(2):107-123. doi: 10.21037/ cdt.2019.12.08 


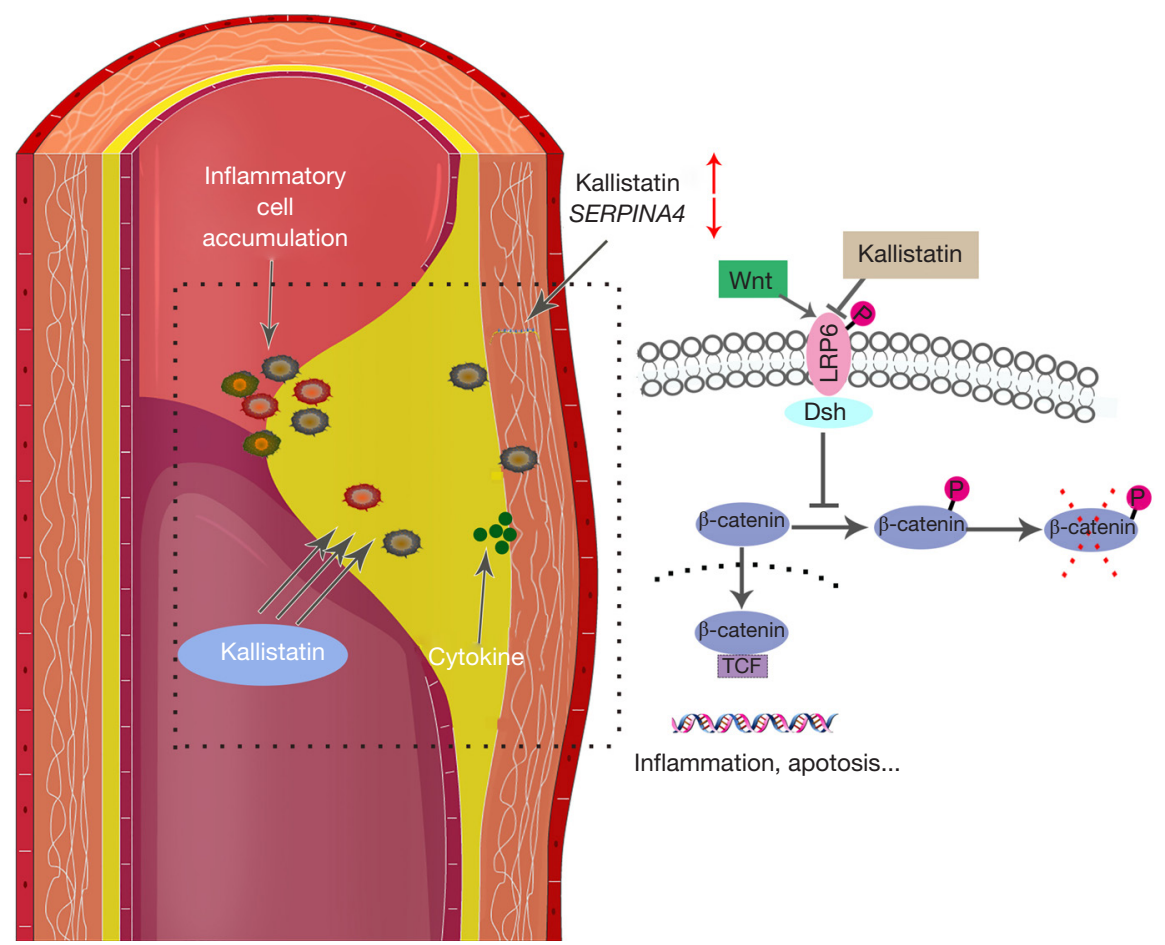

Figure S1 In this study, significant difference of KS (SERPINA4) expression were observed in AAA, compared with the controls. KS (SERPINA4) shows a potential anti-inflammatory role in AAA. Decreased KS (SERPINA4) level is a risk factor of AAA rupture. Treatment with recombination KS suppresses AngII-induced aortic inflammation and Wnt signal pathway. This study highlights the potential therapeutic benefit of KS therapy in preventing aortic aneurysm. KS, kallistatin; AAA, abdominal aortic aneurysm; TCF, T-cell factor.

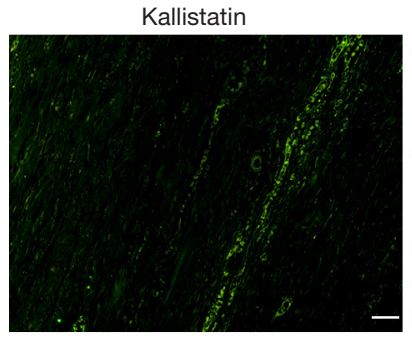

DAPI
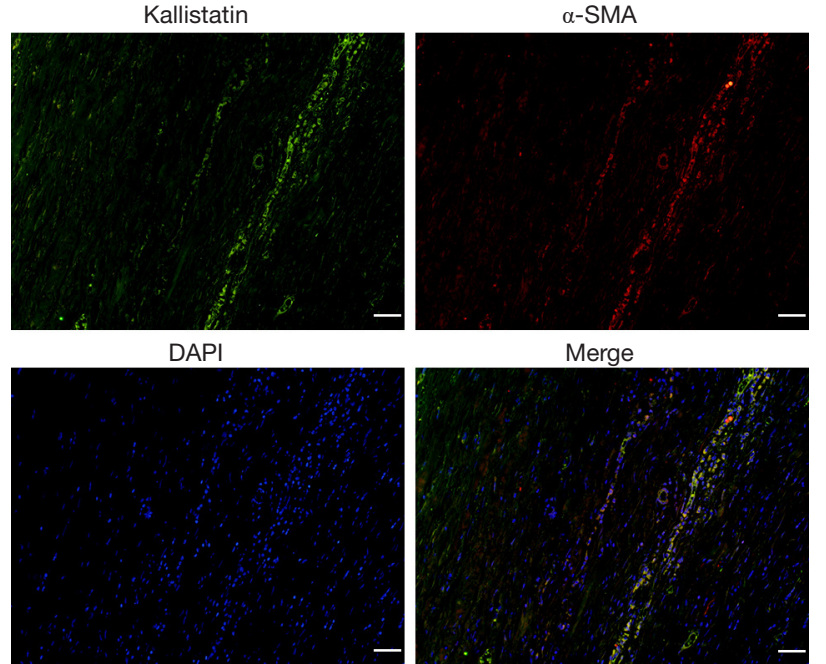

Merge

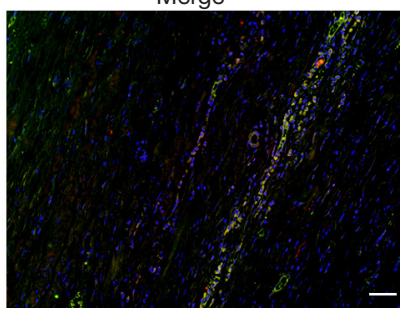

Figure S2 IF staining of the AAA sample. Representative images of IF staining for KS (green) and $\alpha$-SMA (red) in the AAA sample. Scale bar, $50 \mu \mathrm{m}$. IF, immunofluorescence; AAA, abdominal aortic aneurysm; KS, kallistatin; $\alpha$-SMA, alpha-smooth muscle actin.

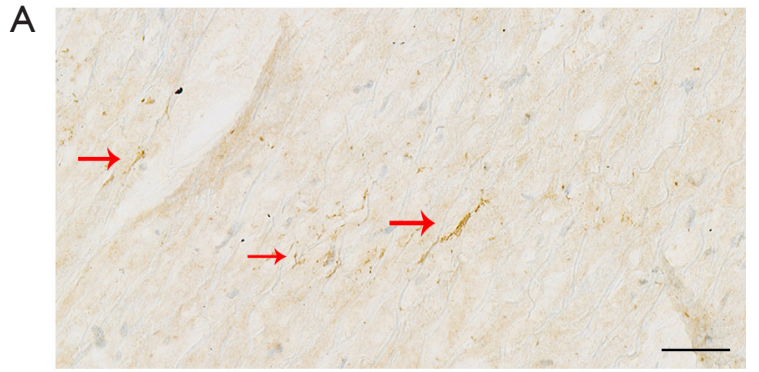

B

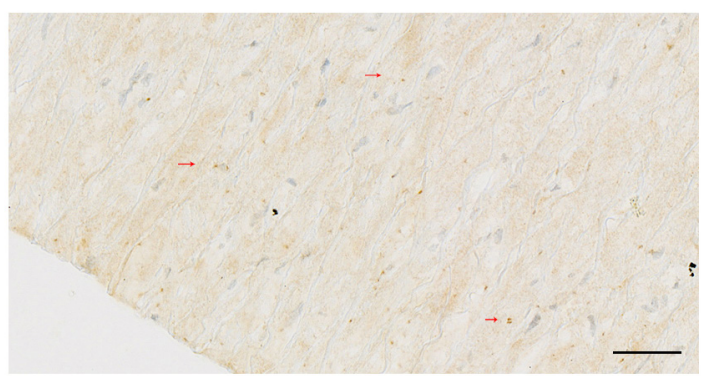

Figure S3 Expression of SERPINA4 mRNA in healthy aortic tissues by in situ hybridization with DIG-labeled SERPINA4 probe. (A) and (B) representative images of in situ hybridization showing the expression of SERPINA4 mRNA (the arrows) in healthy aortic tissue. Scale bar, $50 \mu \mathrm{m}$. DIG, digoxin. 
MHLIDYLLLLLVGLLALSHGQLHVEHDGESCSNSSHQQILETGEGSPSLKIAPANADFAFRFYYLIASETPGK NIFFSPLSISAAYAMLSLGACSHSRSQILEGLGFNLTELSESDVHRGFQHLLHTLNLPGHGLETRVGSALFLS HNLKFLAKFLNDTMAVYEAKLFHTNFYDTVGTIQLINDHVKKETRGKIVDLVSELKKDVLMVLVNYIYFKALW EKPFISSRTTPKDFYVDENTTVRVPMMLQDQEHHWYLHDRYLPCSVLRMDYKGDATVFFILPNQGKMREI EEVLTPEMLMRWNNLLRKRNFYKKLELHLPKFSISGSYVLDQILPRLGFTDLFSKWADLSGITKQQKLEASK SFHKATLDVDEAGTEAAAATSFAIKFFSAQTNRHILRFNRPFLVVIFSTSTQSVLFLGKVVDPTKPAHHHHHH $\mathrm{HHHH}$

\section{SerpinA4h-ZM1}

ATGCATCTTATCGACTACCTGCTCCTCCTGCTGGTTGGACTACTGGCCCTTTCTCATGGCCAGCTGCAC GTTGAGCATGATGGTGAGAGTTGCAGTAACAGCTCCCACCAGCAGATTCTGGAGACAGGTGAGGGCT CCCCCAGCCTCAAGATAGCCCCTGCCAATGCTGACTTTGCCTTCCGCTTCTACTACCTGATCGCTTCG GAGACCCCGGGGAAGAACATCTTTTTCTCCCCGCTGAGCATCTCGGCGGCCTACGCCATGCTTTCCCT GGGGGCCTGCTCACACAGCCGCAGCCAGATCCTTGAGGGCCTGGGCTTCAACCTCACCGAGCTGTC TGAGTCCGATGTCCATAGGGGCTTCCAGCACCTCCTGCACACTCTCAACCTCCCCGGCCATGGGCTG GAAACACGCGTGGGCAGTGCTCTGTTCCTGAGCCACAACCTGAAGTTCCTTGCAAAATTCCTGAATGA CACCATGGCCGTCTATGAGGCTAAACTCTTCCACACCAACTTCTACGACACTGTGGGCACAATCCAGC TTATCAACGACCACGTCAAGAAGGAAACTCGAGGGAAGATTGTGGATTTGGTCAGTGAGCTCAAGAAG GACGTCTTGATGGTGCTGGTGAATTACATTTACTTCAAAGCCCTGTGGGAGAAACCATTCATTTCCTCA AGGACCACTCCCAAAGACTTCTATGTTGATGAGAACACAACAGTCCGGGTGCCCATGATGCTGCAGGA CCAGGAGCATCACTGGTATCTTCATGACAGATACTTGCCCTGCTCGGTGCTACGGATGGATTACAAAGG AGACGCAACCGTGTTTTTCATTCTCCCTAACCAAGGCAAAATGAGGGAGATTGAAGAGGTTCTGACTC CAGAGATGCTAATGAGGTGGAACAACTTGTTGCGGAAGAGGAATTTTTACAAGAAGCTAGAGTTGCATC TTCCCAAGTTCTCCATTTCTGGCTCCTATGTATTAGATCAGATTTTGCCCAGGCTGGGCTTCACGGATCT GTTCTCCAAGTGGGCTGACTTATCCGGCATCACCAAACAGCAAAAACTGGAGGCATCCAAAAGTTTCC ACAAGGCCACCTTGGACGTGGATGAGGCTGGCACCGAGGCTGCAGCAGCCACCAGCTTCGCGATCA AATTCTTCTCTGCCCAGACCAATCGCCACATCCTGCGATTCAACCGGCCCTTCCTTGTGGTGATCTTTT CCACCAGCACCCAGAGTGTCCTCTTTCTGGGCAAGGTCGTCGACCCCACGAAACCAGCTCATCACCA CCATCACCACCATCATCACCATTAA

\begin{tabular}{|c|c|}
\hline $\begin{array}{c}\text { Purity by } \\
\text { SDS-PAGE }\end{array}$ & $0.2 \mu \mathrm{m}$ filtration \\
\hline $85.9 \%$ & Yes \\
\hline
\end{tabular}

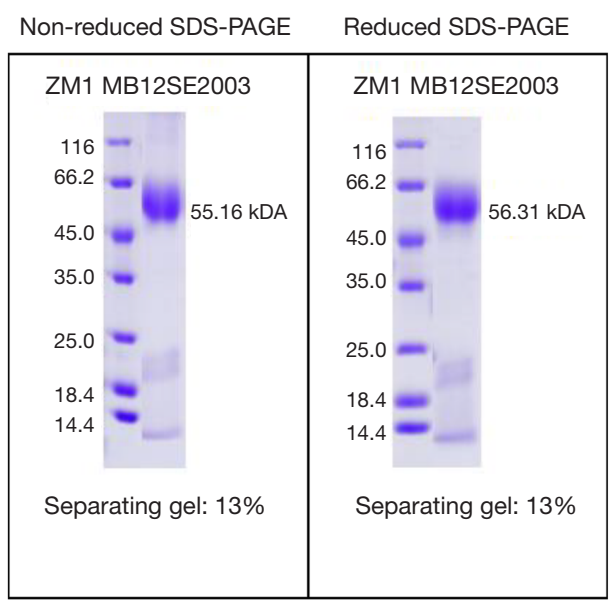

Figure S4 The certification of recombinant kallistatin, which was obtained from Sino Biological Inc. 
A
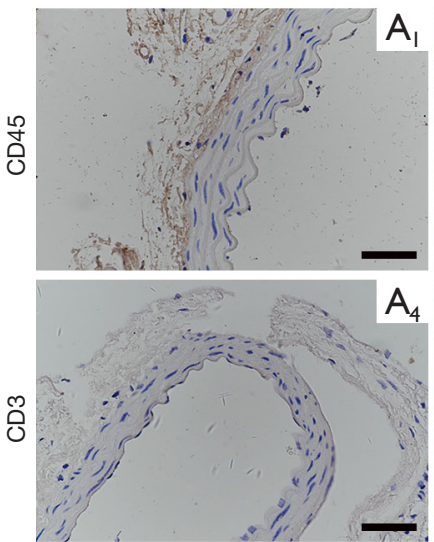

Ang II
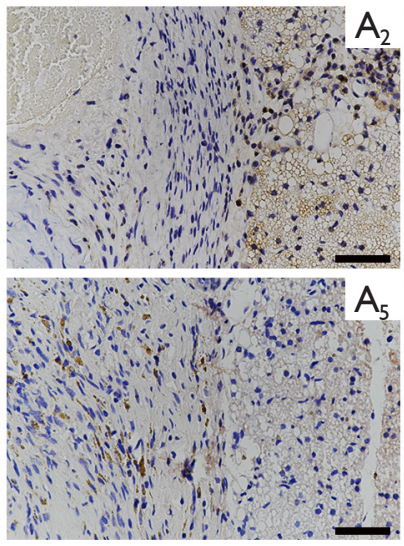

Ang $\mathrm{II}+\mathrm{KS}$
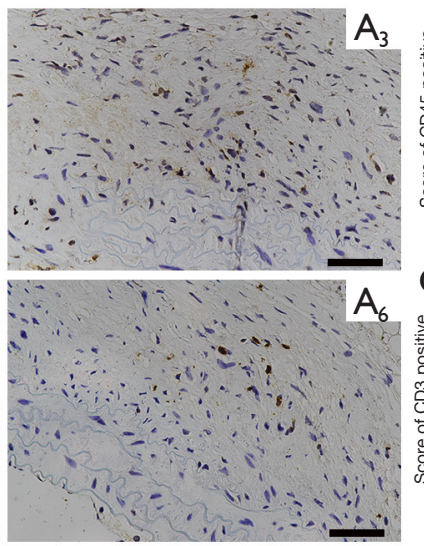

B

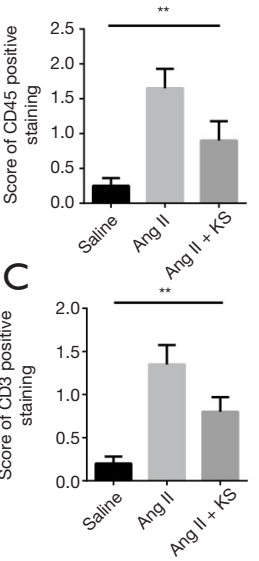

Figure S5 The evaluation of inflammation among the saline group, the AngII group and the AngII + KS group. (A) The representative images of CD45 staining and CD3 staining; (B) semi-quantitative evaluation of CD45 staining and (C) semi-quantitative evaluation of CD3 staining in aorta tissue sample.in aorta tissue sample. Scale bar, $50 \mu \mathrm{m} .{ }^{* *}, \mathrm{P}<0.01$ among the three groups. Data are presented as means \pm SEM. AngII, angiotensin II; KS, kallistatin; SEM, standard error of the mean.

Table S1 The sequences for gene-specific primers

\begin{tabular}{lll}
\hline Gene symbol & Forward primer & Reverse primer \\
\hline SERPINA4 & CCTGCTGGTTGGACTACTGG & CTGTTACTGCAACTCTCACCAT \\
Wnt3 & AGGGCACCTCCACCATTTG & GACACTAACACGCCGAATCA \\
CTNNB1 & AAAGCGGCTGTTAGTCACTGG & CGAGTCATTGCATACTGTCCAT \\
GSK3B & GGCAGCATGAAATTAGCAGA & GGCGACCAGTTCTCCTGAATC \\
ICAM-1 & TTGGGCATAGAGACCCCGTT & GCACATTGCTCAGTTCATACACC \\
VEGFA & AGGGCAGAATCATCACGAAGT & AGGGTCTCGATTGGATGGCA \\
TNF & GAGGCCAAGCCCTGGTATG & CGGGCCGATTGATCTCAGC \\
CD45 & ACCACAAGTTTACTAACGCAAGT & TTTGAGGGGATTCCAGGTAAT \\
CD3 & CCTCTTATCAGTTGGCGTTGG & TTCAGTGACAGGTGATCCTCA \\
MSR1 & GCAGTGGGATCACTTTCACAA & AGCTGTCATTGAGCGAGCATC \\
SMTN & CCCTGGCATCCAAGCGTTT & CTCCACATCGTTCATGGACTC \\
SM-MHC & GGTCACGGTTGGGAAGATGA & GGGCAGGTGTTATAGGGGTT \\
SMemb & GCAGGAGAACACCTAAAGTCTG & TGTCCCGGAATAGGAATATAGCC \\
Coll I & GAGGGCCAAGACGAAGACATC & CAGATCACGTCATCGCACAAC \\
VCAM1 & GGGAAGATGGTCGTGATCCTT & TCTGGGGTGGTCTCGATTTA \\
GAPDH & ACAACTTTGGTATCGTGGAAGG & GCCATCACGCCACAGTTTC \\
\hline
\end{tabular}

